\title{
Stakeholder attitudes to the regulation of traditional and complementary medicine professions: a systematic review
}

Jenny Carè $^{1^{*}}$ (D) Amie Steel ${ }^{1}\left(\mathbb{D}\right.$ and Jon Wardle ${ }^{1,2}$ (D)

\begin{abstract}
Background: There has been a considerable increase in the number of traditional and complementary medicine (T\&CM) practitioners over the past 20 years and in some jurisdictions are estimated to outnumber general practitioners. Despite this globally significant role, it is apparent that worldwide not all T\&CM professions operate under adequate accountability and regulatory oversight for maintaining public protection. To date there has been no published systematic examination of stakeholder opinions regarding regulated and unregulated T\&CM occupations. In response, this review aims to investigate, describe, and analyse attitudes held by a range of stakeholder groups towards the regulation of T\&CM professions.

Methods: A database search of AMED, CINAHL, Embase, Ovid MEDLINE, ProQuest, PsycINFO, PubMed, Scopus, and Google Scholar was conducted for original research published between 2000 and 2020 on stakeholder opinions regarding the regulation of T\&CM professions.
\end{abstract}

Results: Sixty studies across 15 countries reported on the views of six health care stakeholder groups: consumers, T\&CM practitioners, conventional medicine practitioners, professional associations, education providers, and policymakers. Across all stakeholder groups there was between 15\% and 95\% (median 61\%) support for, and 1\% to 57\% (median 14\%) opposition to the regulation of various T\&CM professions. The main reasons for supporting regulation included providing greater public protection, raising training and practice standards, establishing title protection, and gaining acceptance from conventional medicine providers. Concerns regarding regulation included potential restrictions to practice, misappropriation of practice, and medical oversight of T\&CM practitioners. Few studies canvassed the views of professional associations $(n=6)$, education providers $(n=2)$, and policy-makers $(n=2)$.

Conclusions: There appears to be broad support for the regulation of T\&CM professions, although there was wide variation in attitudes as to how this should be applied. Further research, with a particular focus on policy-makers, education providers, and professional associations, is critical to inform appropriate health policy and practice recommendations relating to T\&CM professional regulation across jurisdictions. Systematic review registration: the a priori protocol for this systematic review was registered in PROSPERO and is available at: www.crd.york.ac.uk/PROSPERO/ display_record.asp?ID=CRD42020198767.

*Correspondence: jennifer.m.care@student.uts.edu.au

${ }^{1}$ Australian Research Centre in Complementary and Integrative Medicine,

University of Technology Sydney, Ultimo, NSW 2007, Australia

Full list of author information is available at the end of the article original author(s) and the source, provide a link to the Creative Commons licence, and indicate if changes were made. The images or other third party material in this article are included in the article's Creative Commons licence, unless indicated otherwise in a credit line to the material. If material is not included in the article's Creative Commons licence and your intended use is not permitted by statutory regulation or exceeds the permitted use, you will need to obtain permission directly from the copyright holder. To view a copy of this licence, visit http://creativecommons.org/licenses/by/4.0/. The Creative Commons Public Domain Dedication waiver (http://creativeco mmons.org/publicdomain/zero/1.0/) applies to the data made available in this article, unless otherwise stated in a credit line to the data. 
Keywords: Complementary medicine, Complementary and alternative medicine, Credentialling, Licensure, Metasynthesis, Professionalisation, Regulation, Registration, Systematic review, Traditional and complementary medicine

\section{Background}

Health care systems are coming under increasing pressure from challenges posed by the growing burden of non-communicable diseases [1,2], the health care needs of ageing populations [3], the accelerating incidence of epidemics and pandemics [4], burgeoning health care costs [5], and the prospect of health care workforce shortages [6]. Changing needs, shifting priorities and the increasingly consumer-led nature of health care systems have resulted in significant changes in the contemporary health care workforce. This includes the evolution or growth of new professions and changes in scope of existing professions. According to the World Health Organization (WHO) there are now over 150 occupations in the health workforce sector [7], although one commentator considers this number closer to 350 [8].

One area of the health workforce in which there has been considerable growth and evolution is traditional and complementary medicine ( $\mathrm{T} \& \mathrm{CM})$. $\mathrm{T} \& \mathrm{CM}$ refers to a broad set of health care practices and beliefs indigenous to a culture and place (traditional medicine), and practices that are neither indigenous nor part of the predominant health system of a country (complementary) $[9,10]$. T\&CM designations are therefore jurisdictionally defined [9]. Although some T\&CM practices have ancient roots, the worldwide growth in consumer use and recognition of T\&CM commenced in the latter part of the twentieth century, in part due to the Declaration of Alma-Ata [11] and later the release of WHO traditional medicine strategies $[9,12]$. The rise in T\&CM use has proved to be a global phenomenon, evident in both developing and developed nations [13-20]. Commensurate with this rise in use there has been a substantial increase in the number of T\&CM practitioners over the past 20 years [21-23]. Indeed in some jurisdictions the T\&CM cadre is estimated to outnumber general practitioners (GPs) [23], making this cohort a significant part of the health care workforce globally.

Regulation of health practitioners is generally defined as the actions taken by public authorities to control activities and standards relating to health practice [24-26]. While models of health workforce regulation vary across jurisdictions [27, 28], one schema classifies six categories of occupational licensing: no regulation, self-regulation, state sanctioned self-regulation, statutory self-regulation, co-regulation and statutory regulation [29]. In many jurisdictions the regulation of health professions appears to be moving away from non-government regulatory models towards nationally based regulatory approaches [30], and greater regulatory partnerships between the public, professions, and regulators [27]. Further, the WHO has identified regulation as a key milestone of their global health workforce strategy [6], and regulation of the T\&CM workforce specifically as one of three strategic objectives of the WHO T\&CM strategy [9]. Despite these strategic priorities, there is considerable variation worldwide in the way in which T\&CM occupations are regulated, as well as the form of regulation applied. Regulatory developments for T\&CM practices are argued to be lagging behind their growth in use [31], and not all T\&CM occupations operate within adequate accountability-based, public interest regulatory frameworks [9, 10]. For instance, some $T \& C M$ professions are statutorily regulated in certain countries, others reside outside statutory frameworks but occupy a state-recognised place in health provision, and some T\&CM practices are neither statutorily regulated nor acknowledged by the state, but continue to operate within their jurisdictions, sometimes informally [10].

There is a public health imperative for governments to establish mechanisms for recognising and monitoring $\mathrm{T} \& C M$ practices and practitioners, and promote their appropriate integration or restriction within health care systems [10]. Establishing suitable regulatory frameworks may ensure appropriate and consistent minimum standards of education and practice [10,32], and facilitate workforce mobility across country borders [9], potentially alleviating forecast health care workforce shortages [6], and contributing to the WHO's mission of promoting health for all [33]. WHO, through its traditional medicine strategy, has noted the lack of action in progressing $\mathrm{T} \& \mathrm{CM}$ regulation and encourages member states to engage more actively with this policy to facilitate the appropriate regulation of T\&CM within their jurisdictions [9]. By taking a global perspective the development of insights regarding potential enablers and barriers of regulation across a range of jurisdictions is facilitated, which can inform future application of regulatory policy in a number of different settings.

Despite widespread consumer utilisation of T\&CM, the broadening reach of these practices, and the increasing tendency to regulate $\mathrm{T} \& \mathrm{CM}$ professions, what remains unknown and requires greater understanding are the attitudes and perceptions towards regulation of T\&CM 
across the health care stakeholder landscape. In the broadest sense, stakeholder attitudes are important considerations in many contexts and settings [34]. Within the health care context, understanding stakeholder attitudes is important to ensure that regulation is sustainable, responsive, and appropriate, and serves the public interest in a manner that is reflective of societal norms, expectations, and practices. Attitudes are shaped by self-interest, social identification, and personal values through which opinions are formed [35]. Attitudes and opinions have a bearing on policy by influencing regulatory and policy agendas [35-37]. Disregarding the attitudes of key stakeholders risks privileging the views of certain groups at the expense of others $[36,37]$ and may result in regulatory developments that are not responsive to changing health workforce requirements. To date there has been no systematic examination of stakeholder opinions regarding regulated and unregulated $\mathrm{T} \& C M$ occupations, a deficiency this systematic review aims to address. Consistent with regulatory trends, this review takes an expansive view across a range of stakeholder groups and jurisdictions to investigate, describe, and analyse attitudes towards the regulation of $T \& C M$ professions.

\section{Methods}

\section{Review protocol}

In order to inform the development of evidence-based policy, the objective of this review was to investigate, describe, and analyse all available stakeholder attitudes regarding $T \& C M$ regulation canvassed over the past 20 years, classifying and reporting the data according to emergent stakeholder groupings.

The review protocol was developed in accordance with 'Assessing the Methodological Quality of Systematic Reviews' (AMSTAR) guidelines [38] and the 'Preferred Reporting Items for Systematic Review and Meta-Analysis Protocols' (PRISMA-P) 2015 checklist [39]. It was registered in PROSPERO (\#CRD42020198767) [40] prior to completing the literature search.

\section{Search strategy}

Searches were conducted in eight databases (AMED, CINAHL, Embase, Ovid MEDLINE, ProQuest, PsycINFO, PubMed, Scopus) between 22/05/2020 and $26 / 05 / 2020$, supplemented by a Google Scholar search $26 / 06 / 2020$ to $28 / 06 / 2020$. The search strategy consisted of free text and medical subject heading search terms. T\&CM terms were developed using the Cochrane Complementary Medicine Glossary of CAM terms [41] as well as a selection of commonly used terms within T\&CM professions and practices [42]. Regulation-related terms were developed by the first author (JC) from background reading of research regarding health care regulation [31, 43-45]. Search terms were modified to suit the Google Scholar interface. The research team has published multiple systematic literature reviews related to health policy and $\mathrm{T} \& \mathrm{CM}$, and a librarian was consulted in the development of the database search protocols. Table 1 provides the search terms used for Ovid MEDLINE. The full search protocol is available at: https://www.crd.york.ac. uk/PROSPEROFILES/198767_STRATEGY_20200714. pdf.

\section{Selection criteria}

Studies were included if they were original research, in English, published between 2000 and June 2020. A stakeholder attitude map [34] was used to conceptually consider categories of stakeholders within a health care context. Specific stakeholder groups were not defined a priori. All available stakeholder research canvassing views regarding T\&CM practitioner regulation was accepted for inclusion. From background reading and consideration of stakeholder categories [34] the following groups were expected to feature in the search results: consumers, T\&CM practitioners, conventional medicine practitioners, professional associations, education providers, and policy-makers. Defining T\&CM professions applicable to all jurisdictions was problematic [46, 47], hence this review accepted the classification applied by each included study. Review articles, narrative research, commentaries, editorials, and non-English language studies were excluded.

\section{Study selection}

Retrieved records were imported into EndNote X9 (Clarivate Analytics 2018) by JC. Records were deduplicated, titles and abstracts were screened, and resulting full texts were scrutinised by JC. Those meeting the selection criteria were accepted for inclusion. Reference lists of included manuscripts, and all referenced systematic reviews, were manually searched by JC for additional relevant titles. A proportion of records (10\%) was reviewed at each screening stage by all members of the research team (AS, JC, JW). Any discrepancies regarding inclusion eligibility were resolved through discussion.

\section{Data extraction and appraisal}

A data extraction table was developed in Microsoft Excel $^{\circledR}$ (Microsoft 365) to capture the attributes of interest. The table was established by the research team based on the research aim and informed by previous systematic reviews. It was piloted through the collection of attributes of interest, was developed iteratively, and modified by the research team as data extraction proceeded. Data were recorded by JC from detailed reading of included 
Table 1 Search terms used in Ovid MEDLINE for attitudes to the regulation of traditional and complementary medicine professions

\begin{tabular}{|c|c|c|}
\hline & Traditional and complementary medicine & Regulation \\
\hline 1 & Exp complementary therapies/ & (Accreditation adj20 (profession* OR practitioner)).mp \\
\hline 2 & Complementary medicine.mp & (Certification adj20 (profession* OR practitioner)).mp \\
\hline 3 & Complementary therap*.mp & (Consumer protection adj20 (profession* OR practitioner)).mp \\
\hline 4 & Alternative medicine.mp & Credential?ing.mp \\
\hline 5 & Alternative therap*.mp & Government regulation/ \\
\hline 6 & Natural medicine.mp & Government regulation.mp \\
\hline 7 & Natural therap*.mp & (Healthcare reform adj20 (profession* OR practitioner)).mp \\
\hline 8 & Acupuncture/ & (Health care reform adj20 (profession* OR practitioner)).mp \\
\hline 9 & Acupunctur*.mp & Health care regulation.mp \\
\hline 10 & Aromatherapy/ & Healthcare regulation.mp \\
\hline 11 & Aromatherap*.mp & (Health policy adj20 (profession* OR practitioner)).mp \\
\hline 12 & Ayurved*.mp & Legislation/ \\
\hline 13 & Chiropractic/ & (Legislati* adj20 (profession* OR practitioner)).mp \\
\hline 14 & Manipulation chiropractic/ & Licensure/ \\
\hline 15 & Chiropract*.mp & (Licensure adj20 (profession* OR practitioner)).mp \\
\hline 16 & Herbal medicine/ & Occupation* registration.mp \\
\hline 17 & Herbalis*.mp & Occupation* regulation.mp \\
\hline 18 & Herbal medicine practitioner*.mp & Professionali?ation.mp \\
\hline 19 & Phytotherap*.mp & (Registration adj20 (practitioner OR profession*)).mp \\
\hline 20 & Homeopath*.mp & (Regulation adj20 (practitioner OR profession*)).mp \\
\hline 21 & Homoeopath*.mp & Risk governance.mp \\
\hline 22 & Massage/ & Risk perception.mp \\
\hline 23 & Massage.mp & Risk understanding.mp \\
\hline 24 & Naturopath*.mp & Statutory registration. $\mathrm{mp}$ \\
\hline 25 & Osteopathic physicians/ & Statutory regulation.mp \\
\hline 26 & Osteopathic medicine/ & \\
\hline 27 & Osteopath*.mp & \\
\hline 28 & Exp medicine, east asian traditional/ & \\
\hline 29 & Traditional chinese medicine.mp & \\
\hline 30 & Traditional medicine.mp & \\
\hline 31 & African healing.mp & \\
\hline 32 & African medicine.mp & \\
\hline 33 & Arabic healing.mp & \\
\hline 34 & Arabic medicine.mp & \\
\hline 35 & Indian healing.mp & \\
\hline 36 & Indian medicine.mp & \\
\hline 37 & Japanese medicine.mp & \\
\hline 38 & Japanese healing.mp & \\
\hline 39 & Kampo medicine.mp & \\
\hline 40 & Korean medicine.mp & \\
\hline 41 & Sidda medicine.mp & \\
\hline 42 & Tibetan medicine.mp & \\
\hline 43 & Unani medicine.mp & \\
\hline 44 & Yunani medicine.mp & \\
\hline
\end{tabular}

studies during which the relevant data were transferred to the data table and subsequently verified by the research team.
Identified studies were appraised for risk of bias. Cross-sectional observational studies adopting qualitative research designs were assessed using the Joanna 
Briggs Institute Critical Appraisal Checklist for Qualitative Research [48]. Cross-sectional observational studies employing quantitative research methods were appraised using Hoy et al.s checklist for population-based prevalence studies [49]. The assessments were conducted by JC, and a sample of studies was reviewed by the research team. As this was the first systematic review of this topic, the authors considered it appropriate to include the entirety of available research conducted over the past 20 years irrespective of assessment outcomes.

\section{Data synthesis and analysis}

A meta-analysis was not possible due to significant heterogeneity between studies. Where quantitative data were available this is summarised and narratively analysed. Qualitative data were analysed, categorised inductively, and reported narratively based on themes that emerged from the data in the identified studies. Stakeholder categorisation was undertaken inductively.

Throughout this review, the term regulation refers to the statutory/legislative governance of health care occupations or the registration of practitioners, unless otherwise stated.

\section{Results}

The database, Google Scholar and manual searches yielded 3132 non-duplicated records. Following screening, a total of 54 published and unpublished papers met the inclusion criteria and were selected for review. The reasons for study exclusion are detailed in Fig. 1.

\section{Risk of bias}

Two studies were excluded from critical appraisal because they were qualitative analyses of open-ended questions that were part of larger, and separate, quantitative studies [50,51]. One report included three separate stakeholder studies [52] which were assessed individually. Therefore, a total of 54 studies were appraised.

\section{Cross-sectional observational studies using qualitative research design methods $(n=21)$}

Six studies met the appraisal requirements for the first five domains [53-58]. A further six adequately addressed domain 6 [55-60]. Most studies $(n=16)$ addressed domain 7 [53-70], and all studies met the requirements of the final three domains, except for one which did not address research ethics [63]. The overall risk of bias was considered moderate in 17 studies $[52-55,59,61-$ $72]$ and low in the remaining four [56-58, 60].

See Table 2 for full details of critical appraisal of qualitative studies.

\section{Cross-sectional observational studies using quantitative} research design methods $(n=33)$

Descriptive studies were at the greatest risk of bias in the first four domains where only nine adequately addressed all items [52, 73-80]. All studies met the requirements of domains 5 and 6. Studies performed reasonably well for domains 7 and 8. Domain 9 was considered irrelevant because all studies employed survey methods. Seventeen studies failed to address the final domain [74, 77, 79, 81-94]. Overall, three studies were rated as high risk $[85,91,92], 16$ were considered moderate risk [81, 82, 84, 86-90, 93-100], and 14 studies were judged as low risk [52, 73-80, 83, 101-104].

See Table 3 for full details of critical appraisal of quantitative studies.

\section{Study characteristics}

The 54 included studies consisted of one book chapter [53], two government/industry sponsored reports [52, $75]$, three doctoral/master's theses [58, 78, 94], and 48 journal articles [50, 51, 54-57, 59-77, 79, 81-93, 95104]. Four papers examined two stakeholder groups and were included separately in this review $[70,88,91$, 100]. One report [52], which was published in summarised form [105], consisted of five separate stakeholder studies, two of which were published in their entirety in scholarly journals $[73,101]$. The published version of a third study [80] did not include the full data set relating to regulation. This study and the two remaining studies have been included in this review using the data from the unpublished report [52]. Contact with the corresponding authors of the book chapter, government/ industry reports, and theses confirmed their research has not been published in any journal. Overall, the papers selected for inclusion were 60 separate studies from 54 original publications.

Twenty-four studies employed qualitative design methods, and 36 used quantitative methods. The studies spanned 15 countries: Australia $(n=19)$, Canada $(n=13)$, New Zealand $(n=10)$, UK $(n=5)$, Korea $(n=2)$, USA $(n=2)$, and Egypt, Ghana, India, Netherlands, Portugal, Saudi Arabia, Sierra Leone, South Africa, and Taiwan ( $n=1$ each). Six stakeholder groups were investigated: consumers $(n=8), \mathrm{T} \& C M$ practitioners $(n=19)$, conventional medicine practitioners $(n=23)$, professional associations $(n=6)$, education providers $(n=2)$, and policy-makers $(n=2)$. The T\&CM occupations under study encompassed 36 discrete professions, the most common being naturopathy $(n=26)$, homeopathy $(n=22)$, acupuncture $(n=21)$, herbal medicine $(n=21)$, chiropractic $(n=18)$, and traditional Chinese medicine $(n=13)$. 


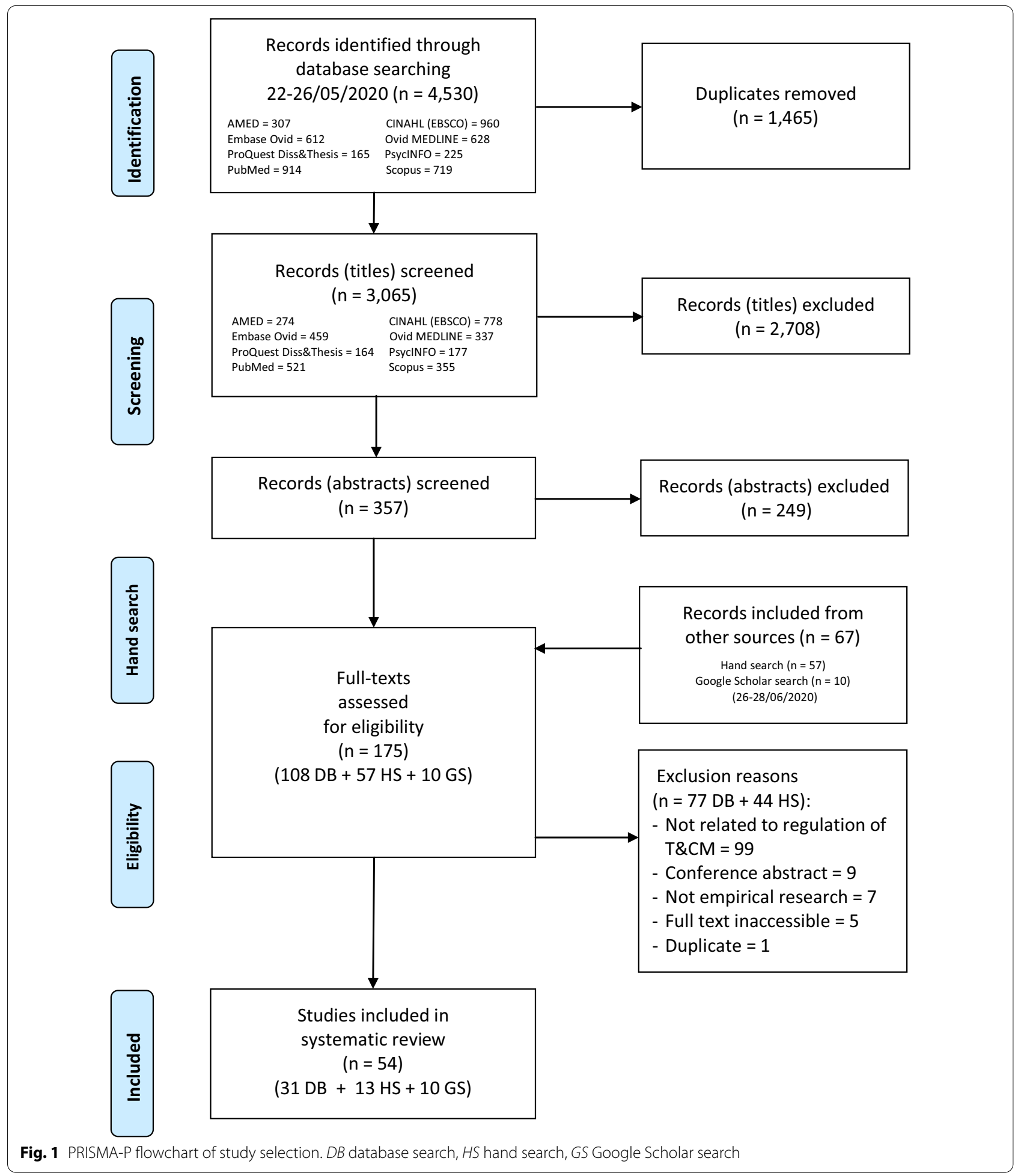

Nine studies did not specify the occupation, using terms such as alternative therapies, complementary and alternative medicine, traditional healing, as well as traditional and complementary medicine.
Table 4 provides details of study characteristics and findings. 
Table 2 Risk of bias assessment for qualitative studies

\begin{tabular}{|c|c|c|c|c|c|c|c|c|c|c|c|}
\hline \multirow[b]{2}{*}{ First author year } & \multicolumn{10}{|c|}{ Domains } & \multirow[b]{2}{*}{ Overall summary (risk) } \\
\hline & 1 & 2 & 3 & 4 & 5 & 6 & 7 & 8 & 9 & 10 & \\
\hline Barnes 2018 [61] & U & $U$ & $U$ & $U$ & $U$ & $\mathrm{~N}$ & Y & Y & Y & Y & Include (moderate) \\
\hline Boon 2004 [53] & Y & Y & Y & Y & Y & $\mathrm{N}$ & Y & Y & Y & Y & Include (moderate) \\
\hline Canaway 2009 [71] & U & $U$ & U & $U$ & U & $\mathrm{N}$ & N & Y & Y & Y & Include (moderate) \\
\hline Cavaco 2017 [72] & U & $U$ & $U$ & U & $U$ & $\mathrm{~N}$ & $\mathrm{~N}$ & Y & Y & Y & Include (moderate) \\
\hline Clarke 2004 [54] & Y & Y & Y & Y & Y & $\mathrm{N}$ & $\mathrm{N}$ & Y & Y & Y & Include (moderate) \\
\hline Ericksen-Pereira 2020 [55] & Y & Y & Y & Y & Y & Y & $\mathrm{N}$ & Y & Y & y & Include (moderate) \\
\hline Flower 2015 [62] & $U$ & $U$ & U & $U$ & $U$ & $\mathrm{~N}$ & Y & Y & Y & Y & Include (moderate) \\
\hline Gilmour 2002 [63] & U & $U$ & U & $U$ & U & $\mathrm{N}$ & Y & Y & $N$ & Y & Include (moderate) \\
\hline Gyasi 2017 [56] & Y & Y & Y & Y & Y & Y & Y & Y & Y & Y & Include (low) \\
\hline James 2020 [59] & U & $U$ & U & U & U & Y & Y & Y & Y & Y & Include (moderate) \\
\hline Jarvis 2015 [64] & U & $U$ & U & $U$ & $U$ & $\mathrm{~N}$ & Y & Y & Y & Y & Include (moderate) \\
\hline Kelly 2005 [65] & U & U & U & $U$ & U & N & Y & Y & Y & Y & Include (moderate) \\
\hline Kelner 2004 [66] & U & $U$ & $U$ & $U$ & U & $\mathrm{N}$ & Y & Y & Y & Y & Include (moderate) \\
\hline Kelner 2004 [67] & U & U & U & U & U & $\mathrm{N}$ & Y & Y & Y & Y & Include (moderate) \\
\hline Kelner 2006 [68] & Y & $U$ & U & $U$ & U & $\mathrm{N}$ & Y & Y & Y & Y & Include (moderate) \\
\hline Lin (Section 9, Hill) 2005 [52] & U & $U$ & U & U & U & $N$ & $\mathrm{~N}$ & Y & Y & Y & Include (moderate) \\
\hline Malhotra 2020 [57] & Y & Y & Y & Y & Y & Y & Y & Y & Y & Y & Include (low) \\
\hline Smith 2015 [58] & Y & Y & Y & Y & Y & Y & Y & Y & Y & Y & Include (low) \\
\hline Steel 2020 [60] & $U$ & $U$ & U & U & U & Y & Y & Y & Y & Y & Include (low) \\
\hline Wardle 2013 [69] & U & $U$ & U & U & U & $\mathrm{N}$ & Y & Y & Y & Y & Include (moderate) \\
\hline Welsh 2004 [70] & $U$ & $U$ & $U$ & U & U & N & Y & Y & Y & Y & Include (moderate) \\
\hline
\end{tabular}

\section{Domains}

1. Is there congruity between the stated philosophical perspective and the research methodology?

2 . Is there congruity between the research methodology and the research question or objectives?

3. Is there congruity between the research methodology and the methods used to collect data?

4. Is there congruity between the research methodology and the representation and analysis of data?

5 . Is there congruity between the research methodology and the interpretation of results?

6. Is there a statement locating the researcher culturally or theoretically?

7. Is the influence of the researcher on the research, and vice-versa, addressed?

8. Are participants, and their voices, adequately represented?

9. Is the research ethical according to current criteria or is there evidence of ethical approval by an appropriate body?

10. Do the conclusions drawn in the research report flow from the analysis, or interpretation, of the data?

$\mathrm{N}=$ criterion not adequately met; $\mathrm{Y}=$ criterion adequately met; $\mathrm{U}=$ unclear if criterion met

\section{Attitudes to regulation}

Fifteen of 24 quantitative studies reported greater than $60 \%$ support for the regulation of $\mathrm{T} \& \mathrm{CM}$ practices. Across all stakeholder groups there was between 15\% and 95\% (median 61\%) support for, and $1 \%$ to $57 \%$ (median $14 \%$ ) opposition to the regulation of various $T \& C M$ professions.

Between $71 \%$ and $95 \%$ of consumers (median $86 \%$ ) were supportive of T\&CM provider regulation [76, 79, $83,91,93,94,102]$, and $1 \%$ to $5 \%$ (median $2 \%$ ) were opposed [76, 79, 102].

T\&CM practitioner support for regulation was between $33 \%$ and $85 \%$ (median 54\%) [74, 82, 92, 96, 97, 100 ], while $8 \%$ to $58 \%$ (median $43 \%$ ) opposed regulation
$[74,82,92,96]$. Notably, the main opposition to regulation came from outlier results in one study of Taiwanese folk medicine practitioners of ba guan, gua sha, reflexology, and tuina whose disapproval of regulation ranged from $42 \%$ to $58 \%$ [92]. Removing these outlier data points from the results shows median T\&CM practitioner opposition to regulation was $25 \%$ [74, 82, 96, 100].

Some $15 \%$ to $92 \%$ (median $66 \%$ ) of conventional medicine providers supported [75, 85-87, 89, 91, 99-101], and $2 \%$ to $49 \%$ (median $11 \%$ ) opposed $[75,85-87,99]$ T\&CM regulation.

Two-thirds of professional association studies [54, $63,68,70]$ reported endorsement for regulation, the only quantitative study finding that $36 \%$ of Australian 
Table 3 Risk of bias assessment for quantitative studies

\begin{tabular}{|c|c|c|c|c|c|c|c|c|c|c|c|}
\hline \multirow[b]{2}{*}{ First author year } & \multicolumn{4}{|c|}{ External validity domains } & \multicolumn{6}{|c|}{ Internal validity domains } & \multirow[b]{2}{*}{ Overall risk } \\
\hline & 1 & 2 & 3 & 4 & 5 & 6 & 7 & 8 & 9 & 10 & \\
\hline Al Mansour 2015 [95] & N & N & N & Y & Y & Y & Y & Y & N/A & Y & Moderate \\
\hline Bensoussan 2004 [73] & Y & Y & Y & Y & Y & Y & Y & Y & N/A & Y & Low \\
\hline Braun 2013 [74] & Y & Y & Y & Y & Y & Y & Y & Y & $\mathrm{N} / \mathrm{A}$ & $\mathrm{N}$ & Low \\
\hline Chaterji 2007 [81] & N & $\mathrm{N}$ & N & $\mathrm{N}$ & Y & Y & Y & Y & N/A & $\mathrm{N}$ & Moderate \\
\hline Cohen 2005 [101] & N & Y & Y & $\mathrm{N}$ & Y & Y & Y & Y & N/A & Y & Low \\
\hline Cottingham 2015 [96] & N & $\mathrm{N}$ & $\mathrm{N}$ & Y & Y & Y & Y & Y & N/A & Y & Moderate \\
\hline Cottingham 2017 [97] & N & $\mathrm{N}$ & N & Y & Y & Y & Y & Y & $\mathrm{N} / \mathrm{A}$ & Y & Moderate \\
\hline Cottingham 2018 [82] & N & $\mathrm{N}$ & N & Y & Y & Y & Y & Y & N/A & $\mathrm{N}$ & Moderate \\
\hline Dooley 2010 [75] & Y & Y & Y & Y & Y & Y & Y & Y & N/A & Y & Low \\
\hline El-Olemy 2014 [102] & N & Y & Y & Y & Y & Y & Y & Y & N/A & Y & Low \\
\hline Emslie 2002 [76] & Y & Y & Y & Y & Y & Y & Y & Y & N/A & Y & Low \\
\hline Evans 2008 [83] & Y & Y & Y & N & Y & Y & N & Y & N/A & $\mathrm{N}$ & Low \\
\hline Flatt 2013 [77] & Y & Y & Y & Y & Y & Y & Y & Y & N/A & $\mathrm{N}$ & Low \\
\hline Hall 2000 [103] & Y & Y & $\mathrm{N}$ & N & Y & Y & Y & Y & N/A & Y & Low \\
\hline Harris 2006 [84] & Y & Y & Y & $N$ & Y & Y & N & Y & N/A & $\mathrm{N}$ & Moderate \\
\hline Langworthy 2000 [85] & N & $\mathrm{N}$ & $\mathrm{N}$ & $N$ & Y & Y & Y & Y & N/A & $\mathrm{N}$ & High \\
\hline $\begin{array}{l}\text { Lin (Section 5, McCabe) } 2005 \\
\text { [52] }\end{array}$ & Y & Y & Y & Y & Y & Y & Y & Y & N/A & Y & Low \\
\hline $\begin{array}{l}\text { Lin (Section 6, McCabe) } 2005 \\
\text { [52] }\end{array}$ & Y & Y & Y & Y & Y & Y & Y & Y & N/A & Y & Low \\
\hline Livingston 2010 [86] & Y & Y & Y & N & Y & Y & Y & Y & N/A & $\mathrm{N}$ & Moderate \\
\hline Montbriand 2000 [87] & N & $\mathrm{N}$ & Y & N & Y & Y & Y & Y & N/A & $\mathrm{N}$ & Moderate \\
\hline Morin 2017 [88] & Y & Y & Y & N & Y & Y & Y & Y & N/A & $\mathrm{N}$ & Moderate \\
\hline Parker 2013 [78] & Y & Y & Y & Y & Y & Y & Y & Y & N/A & Y & Low \\
\hline Poreddi 2016 [104] & Y & $\mathrm{N}$ & Y & Y & Y & Y & Y & Y & N/A & Y & Low \\
\hline Poynton 2006 [98] & Y & Y & N & $\mathrm{N}$ & Y & Y & Y & Y & N/A & Y & Moderate \\
\hline Price 2004 [89] & Y & Y & Y & N & Y & Y & N & Y & N/A & $\mathrm{N}$ & Moderate \\
\hline Semple 2006 [90] & Y & Y & Y & N & Y & Y & Y & Y & N/A & $\mathrm{N}$ & Moderate \\
\hline Taylor 2003 [91] & N & $\mathrm{N}$ & $\mathrm{N}$ & N & Y & Y & N & Y & N/A & $\mathrm{N}$ & High \\
\hline Tiralongo 2010 [99] & Y & Y & Y & $\mathrm{N}$ & Y & Y & Y & $N$ & N/A & Y & Moderate \\
\hline Tsai 2008 [92] & $N$ & $\mathrm{~N}$ & $\mathrm{~N}$ & $N$ & Y & Y & Y & Y & N/A & $\mathrm{N}$ & High \\
\hline Xue 2005 [93] & Y & Y & Y & $N$ & Y & Y & N & Y & N/A & N & Moderate \\
\hline Yu 2015 [100] & $N$ & Y & N & $N$ & Y & Y & Y & N & $\mathrm{N} / \mathrm{A}$ & Y & Moderate \\
\hline Zhang 2006 [94] & Y & Y & $\mathrm{N}$ & Y & Y & Y & Y & Y & N/A & $\mathrm{N}$ & Moderate \\
\hline Zhang 2008 [79] & Y & Y & Y & Y & Y & Y & Y & Y & N/A & $\mathrm{N}$ & Low \\
\hline
\end{tabular}

Domains

1. Was the study's target population a close representation of the national population in relation to relevant variables?

2. Was the sampling frame a true or close representation of the target population?

3. Was some form of random selection used to select the sample, OR was a census undertaken?

4. Was the likelihood of nonresponse bias minimal?

5. Were data collected directly from the subjects (as opposed to a proxy)?

6. Was an acceptable case definition used in the study?

7. Was the study instrument that measured the parameter of interest shown to have validity and reliability?

8. Was the same mode of data collection used for all subjects?

9. Was the length of the shortest prevalence period for the parameter of interest appropriate?

10. Were the numerator(s) and denominator(s) for the parameter of interest appropriate?

$\mathrm{N}=$ criterion not adequately met; $\mathrm{Y}=$ criterion adequately met; $\mathrm{N} / \mathrm{A}=$ criterion not applicable 
Table 4 Study characteristics and attitudes regarding regulation of traditional and complementary medicine professions

\begin{tabular}{|c|c|c|c|c|c|c|}
\hline $\begin{array}{l}\text { First author } \\
\text { year }\end{array}$ & $\begin{array}{l}\text { Country } \\
\text { (jurisdiction, if } \\
\text { limited) }\end{array}$ & Study design & $\begin{array}{l}\text { Stakeholder } \\
\text { population }\end{array}$ & Sample N & T\&CM profession/s examined & Main findings \\
\hline \multicolumn{7}{|c|}{ Consumers ( $n=8)$} \\
\hline $\begin{array}{l}\text { El-Olemy } 2014 \\
{[102]}\end{array}$ & $\begin{array}{l}\text { Egypt (Tanta, } \\
\text { Gharbiya } \\
\text { governorate) }\end{array}$ & $\begin{array}{l}\text { Quantitative } \\
\text { Cross-sectional } \\
\text { self-administered, } \\
\text { supervised ques- } \\
\text { tionnaire survey }\end{array}$ & $\begin{array}{l}\text { Students } \\
\text { (University } \\
\text { School of } \\
\text { Education) }\end{array}$ & 187 & $\begin{array}{l}\text { Traditional and complementary } \\
\text { medicine }\end{array}$ & $\begin{array}{l}\text { 95.2\% agreed that regulating practices } \\
\text { was essential, } 1.6 \% \text { disagreed, 3.2\% were } \\
\text { uncertain } \\
\text { Respondents with previous knowledge of } \\
\text { T\&CM ( } n=146 \text { ) were significantly more pos- } \\
\text { itive towards T\&CM regulation }(p=0.002) \\
95.2 \% \text { agreed that T\&CM practices should be } \\
\text { available and easily accessible, } 92.0 \% \text { agreed } \\
\text { that integration of T\&CM practices into } \\
\text { health care improves patient care }\end{array}$ \\
\hline $\begin{array}{l}\text { Emslie } 2002 \\
\quad \text { [76] }\end{array}$ & $\begin{array}{l}\text { Scotland (Gram- } \\
\text { pian) }\end{array}$ & $\begin{array}{l}\text { Quantitative. } \\
\text { Population-based } \\
\text { postal question- } \\
\text { naire survey }\end{array}$ & Residents & 424 & $\begin{array}{l}\text { Acupuncture, aromatherapy, chi- } \\
\text { ropractic, herbalism, homeopa- } \\
\text { thy, hypnotherapy, osteopathy, } \\
\text { reflexology }\end{array}$ & $\begin{array}{l}90 \% \text { agreed that a register of approved } \\
\text { therapists was essential/desirable (61\% } \\
\text { essential, } 29 \% \text { desirable), } 1 \% \text { agreed it was } \\
\text { unnecessary, } 8 \% \text { gave no opinion } \\
44 \% \text { of } 82 \text { participants indicated concern } \\
\text { about the registration of therapists } \\
65 \% \text { indicated concern about practitioner } \\
\text { qualifications }\end{array}$ \\
\hline Evans 2008 [83] & $\begin{array}{l}\text { New Zealand } \\
\text { (Gisborne) }\end{array}$ & $\begin{array}{l}\text { Quantitative. } \\
\text { Population-based } \\
\text { face to face } \\
\text { questionnaire } \\
\text { survey }\end{array}$ & $\begin{array}{l}\text { Inpatients of } \\
\text { a provincial } \\
\text { hospital }\end{array}$ & 92 & $\begin{array}{l}\text { Acupuncture, antioxidants, } \\
\text { aromatherapy, Bach flower } \\
\text { remedies, Bowen, chiropractic, } \\
\text { colour therapy, detoxification } \\
\text { programs, dietary therapy, } \\
\text { electro/biomagnetic therapy, } \\
\text { herbal therapies, homeopathy, } \\
\text { hypnotherapy, imagery/visu- } \\
\text { alisation, iridology, massage } \\
\text { (Romi Romi), naturopathy, } \\
\text { osteopathy, reflexology, relaxa- } \\
\text { tion techniques, Rongoā Māori, } \\
\text { shark cartilage, spiritual healing, } \\
\text { vitamins, yoga }\end{array}$ & $\begin{array}{l}\text { 78\% agreed T\&CM should be regulated, e.g. } \\
\text { like pharmaceutical drugs, a consultation } \\
\text { with a qualified person first before purchas- } \\
\text { ing medicines } \\
\text { Reasons for supporting regulation were that } \\
\text { medicines could be dangerous, treatment } \\
\text { may not be safe, regulation may give con- } \\
\text { sumers more access to information about } \\
\text { products, and give better informed choice } \\
\text { Reasons for opposing regulation were loss } \\
\text { of freedom of choice, losing control over } \\
\text { one's own health, and would probably make } \\
\text { treatment too expensive }\end{array}$ \\
\hline $\begin{array}{l}\text { Lin } 2005 \text { (Sec- } \\
\text { tion 9, Hill) } \\
\text { [52] (Sum- } \\
\text { marised in } \\
\text { [105]) }\end{array}$ & $\begin{array}{l}\text { Australia (Mel- } \\
\text { bourne) }\end{array}$ & $\begin{array}{l}\text { Qualitative Focus } \\
\text { groups }\end{array}$ & $\begin{array}{l}\text { Consumers } \\
\text { within a } \\
\text { metropolitan } \\
\text { area }\end{array}$ & 24 & $\begin{array}{l}\text { Western herbal medicine, natur- } \\
\text { opathy }\end{array}$ & $\begin{array}{l}\text { The majority of participants indicted there } \\
\text { should be some form of regulation } \\
\text { Because consumers place a great deal of trust } \\
\text { in practitioners, and are often vulnerable, } \\
\text { several participants indicated practition- } \\
\text { ers should have a qualification (implying } \\
\text { an approved qualification) and that they } \\
\text { should be regulated } \\
\text { Those agreeing with regulation said it was } \\
\text { needed to raise the standard of practition- } \\
\text { ers, ensure consistency of care, and stop } \\
\text { unethical practice } \\
\text { Regulation implied recognition of practices } \\
\text { Some were aware that regulation did not } \\
\text { ensure quality care, but thought that it was } \\
\text { important for consumers to know that a } \\
\text { practitioner had undertaken a minimum } \\
\text { standard of training } \\
\text { Concerns included the ability of professional } \\
\text { associations to investigate complaints } \\
\text { against their members, the possibility } \\
\text { that poor practitioners could leave (or be } \\
\text { forced to leave) one association only to join } \\
\text { another, and the need for a body to hear } \\
\text { complaints } \\
\text { A few though regulation would not improve } \\
\text { practitioner quality, and may inhibit them } \\
\text { from trying new treatments, that an intuitive } \\
\text { approach might be lost if practitioners were } \\
\text { required to be registered, and that registra- } \\
\text { tion might restrict what they could practise. } \\
\text { It was believed that regulation would not } \\
\text { address the critical issue of practitioner } \\
\text { communication skills }\end{array}$ \\
\hline
\end{tabular}


Table 4 (continued)

\begin{tabular}{|c|c|c|c|c|c|c|}
\hline $\begin{array}{l}\text { First author } \\
\text { year }\end{array}$ & $\begin{array}{l}\text { Country } \\
\text { (jurisdiction, if } \\
\text { limited) }\end{array}$ & Study design & $\begin{array}{l}\text { Stakeholder } \\
\text { population }\end{array}$ & Sample $N$ & T\&CM profession/s examined & Main findings \\
\hline Taylor 2003 [91] & $\begin{array}{l}\text { New Zealand } \\
\text { (Wanganui) }\end{array}$ & $\begin{array}{l}\text { Quantitative Print- } \\
\text { based question- } \\
\text { naire survey }\end{array}$ & $\begin{array}{l}\text { Consecutive } \\
\text { patients of } \\
\text { general prac- } \\
\text { titioners at } \\
\text { three general } \\
\text { practices }\end{array}$ & 104 & $\begin{array}{l}\text { Acupuncture, aromatherapy, } \\
\text { chiropractic, hypnosis, Rongoā } \\
\text { Māori }\end{array}$ & $\begin{array}{l}\text { 71.1\% agreed regulation of T\&CM practices } \\
\text { should be on a par with orthodox medicine } \\
\text { Patients indicated T\&CM was sometimes, usu- } \\
\text { ally, or always safe } \\
64.4 \% \text { agreed there could be side-effects, and } \\
\text { the majority were aware that there could be } \\
\text { interactions with orthodox medicine }\end{array}$ \\
\hline Xue 2005 [93] & $\begin{array}{l}\text { Australia (Mel- } \\
\text { bourne) }\end{array}$ & $\begin{array}{l}\text { Quantitative Print- } \\
\text { based question- } \\
\text { naire survey. } \\
\text { Convenience } \\
\text { sampling }\end{array}$ & $\begin{array}{l}\text { Members of } \\
\text { the public in } \\
\text { three locali- } \\
\text { ties, pre- } \\
\text { dominantly } \\
\text { Asian and } \\
\text { Caucasian }\end{array}$ & 575 & Chinese medicine & $\begin{array}{l}\text { 76.3\% agreed the practice should be regu- } \\
\text { lated the same as Western medicine } \\
67.7 \% \text { agreed the practice should be more } \\
\text { rigorously regulated } \\
20.9 \% \text { agreed registration improved public } \\
\text { confidence in the practice as a health care } \\
\text { option } \\
\text { 18.7\% agreed registration protected the } \\
\text { public from unqualified service } \\
37.9 \% \text { agreed they would contact the registra- } \\
\text { tion board about service provision concerns } \\
78.2 \% \text { were aware statutory practitioner } \\
\text { registration was recently introduced } \\
29.9 \% \text { agreed they only see a registered } \\
\text { practitioner } \\
80.8 \% \text { agreed they would see a non-registered } \\
\text { practitioner }\end{array}$ \\
\hline $\begin{array}{l}\text { Zhang } 2006 \\
\text { [94] }\end{array}$ & Australia & $\begin{array}{l}\text { Quantitative. } \\
\text { Population-based } \\
\text { random digit } \\
\text { dialled telephone } \\
\text { survey }\end{array}$ & $\begin{array}{l}\text { Representative } \\
\text { proportions } \\
\text { of house- } \\
\text { holds in all } \\
\text { states and } \\
\text { territories }\end{array}$ & 1067 & $\begin{array}{l}\text { Chinese medicine (Chinese herbal } \\
\text { medicine, acupuncture) }\end{array}$ & $\begin{array}{l}\text { 86.4\% agreed with the government regulation } \\
\text { of acupuncture practitioners } \\
\text { 86.0\% agreed that mandatory registration } \\
\text { of acupuncture practitioners provided for } \\
\text { greater public safety and confidence in } \\
\text { acupuncture } \\
85.0 \% \text { agreed with the government regulation } \\
\text { of Chinese herbal medicine practitioners } \\
82.7 \% \text { agreed that mandatory registration } \\
\text { of Chinese herbal medicine practitioners } \\
\text { provided for greater public safety in Chinese } \\
\text { herbal medicine }\end{array}$ \\
\hline $\begin{array}{l}\text { Zhang } 2008 \\
\text { [79] }\end{array}$ & $\begin{array}{l}\text { Australia (Vic- } \\
\text { toria) }\end{array}$ & $\begin{array}{l}\text { Quantitative } \\
\text { Cross-sectional } \\
\text { population-based } \\
\text { random digit } \\
\text { dialled telephone } \\
\text { survey }\end{array}$ & $\begin{array}{l}\text { Households } \\
\text { in eight } \\
\text { geographical } \\
\text { regions }\end{array}$ & 2526 & $\begin{array}{l}\text { Indian herbal medicine (Ayur- } \\
\text { veda), traditional Chinese herbal } \\
\text { medicine, naturopathy, Western } \\
\text { herbal medicine }\end{array}$ & $\begin{array}{l}\text { 89.6\% agreed practitioners should be statuto- } \\
\text { rily regulated (as for medical practitioners), } \\
4.8 \% \text { disagreed, } 5.5 \% \text { were unsure/did not } \\
\text { answer } \\
\text { 46.6\% of herbal medicine users agreed they } \\
\text { were aware of the potential risks of herbal } \\
\text { medicine }\end{array}$ \\
\hline \multicolumn{7}{|c|}{ Traditional and complementary medicine practitioners $(n=19)$} \\
\hline $\begin{array}{c}\text { Bensoussan } \\
2004[73]\end{array}$ & Australia & $\begin{array}{l}\text { Quantitative. } \\
\text { National postal } \\
\text { questionnaire } \\
\text { survey }\end{array}$ & $\begin{array}{l}\text { Naturopaths, } \\
\text { Western } \\
\text { herbal } \\
\text { medicine } \\
\text { practitioners, } \\
\text { homeopaths, } \\
\text { nutritionists }\end{array}$ & 795 & $\begin{array}{l}\text { Western herbal medicine, natur- } \\
\text { opathy }\end{array}$ & $\begin{array}{l}\text { More positive than negative changes were } \\
\text { indicated to result from government regula- } \\
\text { tion for: professional status (78.6\%), practice } \\
\text { standards (73.0\%), education standards } \\
(72.7 \%) \text {, access to research infrastructure } \\
(58.4 \%) \text {, post-graduate education ( } 59.5 \%) \text {, } \\
\text { access to scheduled herbs/products } \\
(55.2 \%) \text {, quality of herbal medicines/prod- } \\
\text { ucts (46.5\%), and establishing occupational } \\
\text { boundaries (41.3\%) } \\
\text { Uncertainty was indicated regarding the } \\
\text { impact of regulation on practitioner income } \\
(56.1 \%), \text { litigation (54.0\%), patient costs } \\
(51.2 \%) \text { and freedom of practice (37.6\%) } \\
\text { Negative impacts were indicated to be more } \\
\text { likely in the area of medical influence on } \\
\text { practice (44.3\%) } \\
44 \% \text { indicated their training poorly prepared } \\
\text { them for inter-professional communication, } \\
22 \% \text { indicated they were poorly prepared in } \\
\text { the area of clinical training }\end{array}$ \\
\hline
\end{tabular}


Table 4 (continued)

\begin{tabular}{|c|c|c|c|c|c|c|}
\hline $\begin{array}{l}\text { First author } \\
\text { year }\end{array}$ & $\begin{array}{l}\text { Country } \\
\text { (jurisdiction, if } \\
\text { limited) }\end{array}$ & Study design & $\begin{array}{l}\text { Stakeholder } \\
\text { population }\end{array}$ & Sample N & T\&CM profession/s examined & Main findings \\
\hline Boon 2004 [53] & $\begin{array}{l}\text { Canada (Toronto, } \\
\text { Ontario) }\end{array}$ & $\begin{array}{l}\text { Qualitative Focus } \\
\text { groups }\end{array}$ & $\begin{array}{l}\text { Acupuncture/ } \\
\text { traditional } \\
\text { Chinese } \\
\text { medicine } \\
\text { practitioners, } \\
\text { homeopaths, } \\
\text { naturopaths }\end{array}$ & 20 & $\begin{array}{l}\text { Acupuncture/traditional Chinese } \\
\text { medicine, homeopathy, } \\
\text { naturopathy }\end{array}$ & $\begin{array}{l}\text { Statutory regulation was identified as the goal } \\
\text { of the professionalisation } \\
\text { All practitioners stated their group was pursu- } \\
\text { ing statutory regulation } \\
\text { Most, but not all, felt this was an important } \\
\text { goal for their occupation } \\
\text { Many considered regulation would result in } \\
\text { some form of monopoly for their practice } \\
\text { Regulation would prevent the co-optation } \\
\text { of their skills and knowledge, allow the } \\
\text { achievement of social closure by establish- } \\
\text { ing education and qualifications standards } \\
\text { that would prevent co-optation by those } \\
\text { outside the profession } \\
\text { Attempts at closure were hampered by lack } \\
\text { of internal cohesion, and disagreement } \\
\text { over the content and form of education } \\
\text { and practice standards, particularly for } \\
\text { homeopathy and acupuncture/traditional } \\
\text { Chinese medicine professions } \\
\text { Fragmentation was partially due to the } \\
\text { intra-professional diversity of practices and } \\
\text { philosophies } \\
\text { Some feared a loss of freedom to practise due } \\
\text { to regulation } \\
\text { In T\&CM finding a place within the health care } \\
\text { system, the public was one of their biggest } \\
\text { allies } \\
\text { Some homeopaths and naturopaths recast } \\
\text { their work as possibly harmful in order to be } \\
\text { eligible for regulation }\end{array}$ \\
\hline Braun 2013 [74] & Australia & $\begin{array}{l}\text { Quantitative Online } \\
\text { questionnaire } \\
\text { survey }\end{array}$ & $\begin{array}{l}\text { Naturopaths, } \\
\text { Western } \\
\text { herbal } \\
\text { medicine } \\
\text { practitioners }\end{array}$ & 479 & $\begin{array}{l}\text { Western herbal medicine, natur- } \\
\text { opathy }\end{array}$ & $\begin{array}{l}\text { 85\% agreed practitioners should be formally } \\
\text { registered to safeguard the public, } 8 \% \\
\text { disagreed, } 8 \% \text { were unsure } \\
\text { Responses indicated possible benefits of } \\
\text { regulation were increased public safety, } \\
\text { protection from inadequately trained prac- } \\
\text { titioners within and outside their profession, } \\
\text { increased practice standards, increased } \\
\text { confidence and credibility of the profession } \\
\text { and its broader integration into the health } \\
\text { care system } \\
\text { Some ( } n=9 \text { ) who agreed with regulation did } \\
\text { not agree that public safety was the main } \\
\text { issue, stating that being a member of a } \\
\text { professional association was the same as } \\
\text { registration so formal registration was not } \\
\text { necessary } \\
\text { Others ( } n=9 \text { ) expressed concern that } \\
\text { practitioners outside their profession could } \\
\text { regulate their profession if formal registra- } \\
\text { tion occurred }\end{array}$ \\
\hline
\end{tabular}


Table 4 (continued)

\begin{tabular}{|c|c|c|c|c|c|}
\hline $\begin{array}{l}\text { First author } \\
\text { year }\end{array}$ & $\begin{array}{l}\text { Country } \\
\text { (jurisdiction, if } \\
\text { limited) }\end{array}$ & Study design & $\begin{array}{l}\text { Stakeholder } \\
\text { population }\end{array}$ & Sample N & T\&CM profession/s examined \\
\hline $\begin{array}{l}\text { Canaway } 2009 \\
\text { [71] }\end{array}$ & $\begin{array}{l}\text { Australia (Mel- } \\
\text { bourne) }\end{array}$ & $\begin{array}{l}\text { Qualitative Semi- } \\
\text { structured inter- } \\
\text { views Snowball } \\
\text { sampling }\end{array}$ & $\begin{array}{l}\text { Naturopaths } \\
\text { (senior } \\
\text { practitioners } \\
\text { mostly in } \\
\text { leadership } \\
\text { roles) }\end{array}$ & 7 & Naturopathy \\
\hline
\end{tabular}


Table 4 (continued)

\begin{tabular}{|c|c|c|c|c|c|c|}
\hline $\begin{array}{l}\text { First author } \\
\text { year }\end{array}$ & $\begin{array}{l}\text { Country } \\
\text { (jurisdiction, if } \\
\text { limited) }\end{array}$ & Study design & $\begin{array}{l}\text { Stakeholder } \\
\text { population }\end{array}$ & Sample N & T\&CM profession/s examined & Main findings \\
\hline $\begin{array}{l}\text { Cottingham } \\
2018[82]\end{array}$ & New Zealand & $\begin{array}{l}\text { Quantitative Online } \\
\text { questionnaire } \\
\text { survey. Conveni- } \\
\text { ence sampling }\end{array}$ & $\begin{array}{l}\text { Registered } \\
\text { massage } \\
\text { therapists }\end{array}$ & 104 & Massage & $\begin{array}{l}56 \% \text { supported statutory registration. } .^{\text {a }} \\
93 \% \text { supported registration, of which } 67 \% \\
\text { supported statutory registration, 31\% sup- } \\
\text { ported voluntary registration, } 1 \% \text { preferred } \\
\text { other registration types (not-specified) }\end{array}$ \\
\hline $\begin{array}{l}\text { Ericksen-Pereira } \\
2020[55]\end{array}$ & South Africa & $\begin{array}{l}\text { Qualitative Emailed } \\
\text { unstructured } \\
\text { (open-ended) } \\
\text { survey or face to } \\
\text { face unstructured } \\
\text { interviews }\end{array}$ & Naturopaths & 21 & Naturopathy & $\begin{array}{l}\text { Registration was important to all participants } \\
\text { because it allowed them to use the title of } \\
\text { naturopath and practise legally } \\
\text { Challenges of registration created impedi- } \\
\text { ments to establishing viable practices, } \\
\text { including being deregistered when failing } \\
\text { to pay on time, bureaucracy requiring fur- } \\
\text { ther application fees and increased annual } \\
\text { re-registering fees, the need to register } \\
\text { within the first six months of graduating or } \\
\text { undergo a competency assessment requir- } \\
\text { ing a fee, annual registration fees regardless } \\
\text { of whether graduates were in a financial } \\
\text { position to establish a practice, costs of } \\
\text { registration (around } 20 \% \text { of the average } \\
\text { salary of a new graduate), being prevented } \\
\text { from sharing clinic space or working in } \\
\text { interdisciplinary practices with registered } \\
\text { practitioners such as medical doctors which } \\
\text { prevented integrative practices }\end{array}$ \\
\hline Flatt 2013 [71] & New Zealand & $\begin{array}{l}\text { Quantitative Postal } \\
\text { questionnaire } \\
\text { survey }\end{array}$ & $\begin{array}{l}\text { Naturopaths } \\
\text { and Western } \\
\text { herbal } \\
\text { medicine } \\
\text { practitioners }\end{array}$ & 120 & $\begin{array}{l}\text { Western herbal medicine, natur- } \\
\text { opathy }\end{array}$ & $\begin{array}{l}\text { Over } 60 \% \text { agreed that change of regula- } \\
\text { tory status would have positive effects on } \\
\text { professional status, professional relation- } \\
\text { ships, integrative practice, potential health } \\
\text { subsidies, practitioner competence, and } \\
\text { practice and education standards } \\
\text { Over } 50 \% \text { agreed that change of regulatory } \\
\text { status would have positive effects on pro- } \\
\text { fessional equity, shared care, conduct and } \\
\text { discipline and continuing education } \\
\text { Almost } 60 \% \text { disagreed that regulatory change } \\
\text { would have a positive effect on associa- } \\
\text { tion fees } \\
\text { Just under } 50 \% \text { felt there would be negative } \\
\text { effects on freedom of practice } \\
\text { Around } 50 \% \text { were uncertain about the effects } \\
\text { on income, litigation, research access, bio- } \\
\text { medical influence and career prospects } \\
\text { There was little agreement on the impact on } \\
\text { occupational boundaries, access and quality } \\
\text { of medicines, patient numbers, patient well- } \\
\text { being, and patient access }\end{array}$ \\
\hline Gyasi 2017 [56] & $\begin{array}{l}\text { Ghana (Ashanti } \\
\text { Region) }\end{array}$ & $\begin{array}{l}\text { Qualitative In-depth } \\
\text { interviews }\end{array}$ & $\begin{array}{l}\text { Traditional } \\
\text { healers }\end{array}$ & 7 & Traditional healing & $\begin{array}{l}\text { Despite being available, most practitioners } \\
\text { were not registered and practised without } \\
\text { any regulatory oversight } \\
\text { Most registered practitioners were located } \\
\text { in urban areas, where there was greater } \\
\text { competition and need to uphold ethical } \\
\text { and quality standards } \\
\text { Registered practitioners thought registration } \\
\text { was important to assure consumers of the } \\
\text { quality of medicines sold and for the prepa- } \\
\text { ration of herbal prescriptions } \\
\text { Reasons for not registering include difficulties } \\
\text { such as having to travel to town to register, } \\
\text { the time and cost involved, the complexity } \\
\text { and stress, and the lack of information about } \\
\text { how to register } \\
\text { Non-registered practitioners were ready to } \\
\text { register given the opportunity }\end{array}$ \\
\hline
\end{tabular}


Table 4 (continued)

\begin{tabular}{|c|c|c|c|c|c|c|}
\hline $\begin{array}{l}\text { First author } \\
\text { year }\end{array}$ & $\begin{array}{l}\text { Country } \\
\text { (jurisdiction, if } \\
\text { limited) }\end{array}$ & Study design & $\begin{array}{l}\text { Stakeholder } \\
\text { population }\end{array}$ & Sample N & T\&CM profession/s examined & Main findings \\
\hline ljaz 2015 [50] & Canada (Ontario) & $\begin{array}{l}\text { Qualitative Online } \\
\text { and postal } \\
\text { questionnaire } \\
\text { survey analysis } \\
\text { of open-ended } \\
\text { question }\end{array}$ & $\begin{array}{l}\text { Chinese } \\
\text { medicine } \\
\text { practitioners, } \\
\text { homeopaths, } \\
\text { naturopaths }\end{array}$ & 688 & $\begin{array}{l}\text { Acupuncture/Chinese medicine, } \\
\text { homeopathy, naturopathy }\end{array}$ & $\begin{array}{l}\text { The majority supported regulation but } 33 \% \\
\text { of Chinese medicine practitioners, } 44 \% \text { of } \\
\text { homeopaths and } 48 \% \text { of naturopaths were } \\
\text { concerned about regulation } \\
\text { Concerns related to the way regulation was } \\
\text { being implemented, and whether the regu- } \\
\text { lating body overseeing the implementation } \\
\text { was representative of the profession } \\
\text { A number were concerned about unwanted } \\
\text { financial and administrative burdens on } \\
\text { practitioners, and costs being passed onto } \\
\text { patients } \\
\text { Concerns regarding regulation were that } \\
\text { regulation would reduce or restrict practice } \\
\text { scopes, or would not expand adequately to } \\
\text { allow for diagnostic rights and biomedical } \\
\text { testing, inter-occupational overlapping } \\
\text { practice scopes, co-optation of practice, } \\
\text { including co-optation from other T\&CM } \\
\text { professions (e.g. between homeopaths } \\
\text { and naturopaths), inappropriate or unfair } \\
\text { registration standards, e.g. how regulation } \\
\text { would assess practitioner qualifications, } \\
\text { grandparenting provisions, and language } \\
\text { proficiency requirements, some practition- } \\
\text { ers may be inappropriately excluded from } \\
\text { registration, and that regulatory changes } \\
\text { threatened underlying paradigmatic foun- } \\
\text { dations of practice } \\
\text { Ase of } \\
\text { rather, there should were concerned that practice would } \\
\text { become 'medicalized', and that regulators } \\
\text { ibilicense } \\
\text { did not adequately take into account the } \\
\text { 'culture and tradition' of practices } \\
\text { Some considered that training of some practi- } \\
\text { tioners was inadequate } \\
\text { likely improve quality of patient care by } \\
\text { raising practitioner level of treatment, } \\
\text { and increased biomedical training would } \\
\text { improve competency and enhance cred- } \\
\end{array}$ \\
\hline $\begin{array}{l}\text { Malhotra } 2020 \\
\text { [57] }\end{array}$ & Australia & $\begin{array}{l}\text { Qualitative } \\
\text { Semi-structured } \\
\text { telephone inter- } \\
\text { views }\end{array}$ & Naturopaths & 20 & Naturopathy & $\begin{array}{l}\text { A primary barrier to implementing integrated } \\
\text { care models was the lack of acknowl- } \\
\text { edgment from conventional medical } \\
\text { practitioners } \\
\text { Conventional doctors often had concerns } \\
\text { regarding the efficacy of naturopathic } \\
\text { treatments } \\
\text { The current self-regulation model and } \\
\text { absence of statutory regulation was seen as } \\
\text { a barrier to legitimising the profession } \\
\text { The lack of a well-defined curriculum and } \\
\text { standard training competencies framework } \\
\text { was considered a barrier to being acknowl- } \\
\text { edged by conventional medical practition- } \\
\text { ers as a legitimate profession }\end{array}$ \\
\hline
\end{tabular}


Table 4 (continued)

\begin{tabular}{|c|c|c|c|c|c|c|}
\hline $\begin{array}{l}\text { First author } \\
\text { year }\end{array}$ & $\begin{array}{l}\text { Country } \\
\text { (jurisdiction, if } \\
\text { limited) }\end{array}$ & Study design & $\begin{array}{l}\text { Stakeholder } \\
\text { population }\end{array}$ & Sample N & T\&CM profession/s examined & Main findings \\
\hline Morin 2017 [88] & $\begin{array}{l}\text { Canada (Que- } \\
\text { bec) }\end{array}$ & $\begin{array}{l}\text { Quantitative Postal } \\
\text { questionnaire } \\
\text { survey }\end{array}$ & Osteopaths & 297 & Osteopathy & $\begin{array}{l}62 \% \text { would be moderately/greatly influenced } \\
\text { by government regulation and the estab- } \\
\text { lishment of university-based programs, 38\% } \\
\text { would not be very influenced/influenced } \\
\text { by regulation } \\
\text { Practitioners indicated regulation would } \\
\text { promote collaboration, } 97.2 \% \text { agreeing that } \\
\text { collaborating with physicians was slightly/ } \\
\text { quite/very important }\end{array}$ \\
\hline Parker 2013 [78] & Canada (Ontario) & $\begin{array}{l}\text { Quantitative Cross- } \\
\text { sectional online } \\
\text { questionnaire } \\
\text { survey }\end{array}$ & Homeopaths & 329 & Homeopathy & $\begin{array}{l}\text { Most supported regulation (mean agreement } \\
\text { score 3.9 (SD 1.2), } 5 \text { point Likert scale, } \\
n=273 \text { ) } \\
\text { Most felt regulation would: improve credibility } \\
\text { with the public ( } 4.3 \text { (SD 1.0), } n=275 \text { ) and } \\
\text { other health care professionals (4.1 (SD 1.1), } \\
n=274 \text { ), benefit the public ( } 4.0 \text { (SD 1.2), } \\
n=275), \text { improve quality of patient care ( } 3.8 \\
\text { (SD 1.2), } n=275 \text { ), and benefit homeopaths } \\
\text { (3.6 (SD1.3), } n=273 \text { ) } \\
\text { There was less certainty regarding the impact } \\
\text { on practice (2.9 (1.4), } n=270 \text { ) } \\
\text { 70\% intended to apply for registration but } \\
35.9 \% \text { appeared ineligible due to not } \\
\text { meeting educational or grandparenting } \\
\text { requirements } \\
\text { Those that appeared both eligible and ineli- } \\
\text { gible for registration generally supported } \\
\text { regulation } \\
\text { Those potentially ineligible were older, had } \\
\text { been practising for longer, were more likely } \\
\text { to work in a home based office, appeared } \\
\text { to spend less time with patients on initial } \\
\text { visit, and were less likely to hold a college/ } \\
\text { university degree }\end{array}$ \\
\hline Smith 2015 [58] & New Zealand & $\begin{array}{l}\text { Qualitative } \\
\text { Semi-structured } \\
\text { interviews }\end{array}$ & $\begin{array}{l}\text { Massage } \\
\text { educators, } \\
\text { therapists, } \\
\text { and students }\end{array}$ & 20 & Massage & $\begin{array}{l}\text { Practitioners supported both government } \\
\text { regulation and regulation by the profes- } \\
\text { sional association. Some were opposed to } \\
\text { compulsory statutory regulation } \\
\text { Regulation was viewed as a useful step to } \\
\text { move the profession towards legitimation } \\
\text { and professional recognition } \\
\text { Both statutory and self-regulation were identi- } \\
\text { fied as mechanisms to control practice, } \\
\text { establish a scope of practice, achieve recog- } \\
\text { nition, access government funding, achieve } \\
\text { consistency, facilitate professionalism, and } \\
\text { establish standard education levels } \\
\text { Practitioners stressed the need for clear } \\
\text { scopes of practice } \\
\text { Education required a cohesive set of standards } \\
\text { across education providers } \\
\text { Issues regarding regulation were cost, being } \\
\text { valued as a profession, government regula- } \\
\text { tion would establish standards of education, } \\
\text { professional association has standards and } \\
\text { rules } \\
\text { Some thought degree level education should } \\
\text { be the benchmark, others thought there } \\
\text { was room for both diploma and degree } \\
\text { levels as long as scopes were clear }\end{array}$ \\
\hline
\end{tabular}


Table 4 (continued)

\begin{tabular}{|c|c|c|c|c|c|c|}
\hline $\begin{array}{l}\text { First author } \\
\text { year }\end{array}$ & $\begin{array}{l}\text { Country } \\
\text { (jurisdiction, if } \\
\text { limited) }\end{array}$ & Study design & $\begin{array}{l}\text { Stakeholder } \\
\text { population }\end{array}$ & Sample N & T\&CM profession/s examined & Main findings \\
\hline Steel 2020 [60] & $\begin{array}{l}\text { Australia } \\
\text { (Queensland) }\end{array}$ & $\begin{array}{l}\text { Qualitative } \\
\text { Semi-structured } \\
\text { interviews }\end{array}$ & $\begin{array}{l}\text { Comple- } \\
\text { mentary } \\
\text { medicine } \\
\text { practitioners } \\
\text { specialising } \\
\text { in maternity } \\
\text { care (acu- } \\
\text { puncturists, } \\
\text { doulas, chi- } \\
\text { ropractors, } \\
\text { massage } \\
\text { therapists, } \\
\text { naturopaths, } \\
\text { osteopaths) }\end{array}$ & 23 & $\begin{array}{l}\text { Acupuncture, chiropractic, } \\
\text { doula, massage, naturopathy, } \\
\text { osteopathy }\end{array}$ & $\begin{array}{l}\text { Regulation was needed to gain increased } \\
\text { respect from other professions and because } \\
\text { of concerns about unethical financial prac- } \\
\text { tice such as profiteering by practitioners } \\
\text { Education standards were a concern for some } \\
\text { participants due to } \\
\text { a lack of consistency across institutions and } \\
\text { qualifications } \\
\text { Education standards was also perceived by } \\
\text { some practitioners to impact the ability of } \\
\text { other health professions to work alongside } \\
\text { them, some practitioners perceiving that } \\
\text { inconsistency in training created a nega- } \\
\text { tive perception of their profession among } \\
\text { conventional providers } \\
\text { Often there was disagreement about the } \\
\text { impact of regulation, however there was } \\
\text { agreement from both regulated and } \\
\text { unregulated professions that regulation was } \\
\text { tied to improved training standards } \\
\text { Regulation was not always viewed favourably } \\
\text { by participants due to the perception of } \\
\text { practice limits being imposed } \\
\text { Some suggested the absence of regulation } \\
\text { created difficulties in referring peers to } \\
\text { appropriate colleges of education }\end{array}$ \\
\hline Tsai 2008 [92] & Taiwan (Taipei) & $\begin{array}{l}\text { Quantitative } \\
\text { Print-based } \\
\text { cross-sectional } \\
\text { questionnaire } \\
\text { survey }\end{array}$ & $\begin{array}{l}\text { Folk medicine } \\
\text { practitioners }\end{array}$ & 400 & $\begin{array}{l}\text { Ba guan, gua sha, reflexology, } \\
\text { tuina }\end{array}$ & $\begin{array}{l}\text { Around one half of all respondents agreed } \\
\text { that statutory regulation was necessary for } \\
\text { all practices } \\
64 \%-85 \% \text { agreed credentialling of folk medi- } \\
\text { cine practitioners was necessary } \\
65 \%-82 \% \text { agreed practitioners should receive } \\
\text { formal education/training } \\
\text { Support for regulation: } \\
56.9 \% \text { of city versus } 50.8 \% \text { of country ba guan } \\
\text { practitioners ( } p=0.26) \\
52.8 \% / 42.1 \% \text { city/country gua sha practition- } \\
\text { ers }(p=0.04) \\
53.8 \% / 42.7 \% \text { city/country reflexology practi- } \\
\text { tioners }(p=0.04) \\
58.4 \% / 58.5 \% \text { city/country tuina practitioners } \\
(p=1.0) \\
\text { Opposition to regulation: } \\
43.1 \% / 49.2 \% \text { city/country ba guan practition- } \\
\text { ers }(p=0.26) \\
47.2 \% / 57.9 \% \text { city/country gua sha practition- } \\
\text { ers }(p=0.04) \\
46.2 \% / 57.3 \% \text { city/country reflexology practi- } \\
\text { tioners }(p=0.04) \\
41.6 \% / 41.5 \% \text { of city/country tuina practition- } \\
\text { ers }(p=1.0)\end{array}$ \\
\hline $\begin{array}{l}\text { Wardle } 2013 \\
\text { [69] }\end{array}$ & $\begin{array}{l}\text { Australia (Darling } \\
\text { Downs) }\end{array}$ & $\begin{array}{l}\text { Qualitative } \\
\text { Semi-structured } \\
\text { interviews }\end{array}$ & Naturopaths & 20 & Naturopathy & $\begin{array}{l}\text { Regulation was considered positive by all but } \\
\text { one practitioner } \\
\text { Regulation was seen by many practitioners as } \\
\text { the solution to many of the problems of the } \\
\text { profession } \\
\text { The primary reason for supporting regulation } \\
\text { was to rid the profession of unethical, } \\
\text { bogus, or fraudulent individuals who were } \\
\text { practising without the required qualifica- } \\
\text { tions } \\
\text { Regulation would overcome the challenges } \\
\text { of increasing external influences, internal } \\
\text { division/fragmentation, professional accept- } \\
\text { ance, problems in education of practition- } \\
\text { ers, and co-optation by undertrained/ } \\
\text { fraudulent/practitioners that devalued the } \\
\text { profession }\end{array}$ \\
\hline
\end{tabular}


Table 4 (continued)

\begin{tabular}{|c|c|c|c|c|c|c|}
\hline $\begin{array}{l}\text { First author } \\
\text { year }\end{array}$ & $\begin{array}{l}\text { Country } \\
\text { (jurisdiction, if } \\
\text { limited) }\end{array}$ & Study design & $\begin{array}{l}\text { Stakeholder } \\
\text { population }\end{array}$ & Sample N & T\&CM profession/s examined & Main findings \\
\hline Yu 2015 [100] & Korea & $\begin{array}{l}\text { Quantitative Email, } \\
\text { postal and print- } \\
\text { based question- } \\
\text { naire survey }\end{array}$ & $\begin{array}{l}\text { Acupuncturists, } \\
\text { alternative } \\
\text { therapists, } \\
\text { chiroprac- } \\
\text { tors, clinical } \\
\text { art therapists, } \\
\text { feet massage } \\
\text { therapists, } \\
\text { laughter } \\
\text { therapists, } \\
\text { Qi gong } \\
\text { therapists } \\
\text { and trainees } \\
\text { of traditional } \\
\text { Chinese } \\
\text { medicine }\end{array}$ & 62 & $\begin{array}{l}\text { Acupuncture, alternative medi- } \\
\text { cine, chiropractic, clinical art, } \\
\text { feet massage, laughter, Qi gong, } \\
\text { trainees of traditional Chinese } \\
\text { medicine }\end{array}$ & $\begin{array}{l}\text { Across all provider groups, medical and T\&CM } \\
\text { professionals, } 32.8 \% \text { agreed legislation to } \\
\text { govern providers was preferable, } 25.0 \% \\
\text { agreed with qualifications/accreditation, } \\
13.6 \% \text { agreed with making/evaluating } \\
\text { standards } \\
\text { 48.4\% agreed that a college/university quali- } \\
\text { fication was necessary for gaining qualifica- } \\
\text { tions, } 22.6 \% \text { agreed with taking and passing } \\
\text { a government administered examination } \\
53.3 \% \text { agreed legislation and national control } \\
\text { for management of qualifications was } \\
\text { preferable, } 29.0 \% \text { agreed that a national } \\
\text { examination was preferable }\end{array}$ \\
\hline \multicolumn{7}{|c|}{ Conventional medicine practitioners $(n=23)$} \\
\hline $\begin{array}{r}\text { Al Mansour } \\
2015 \text { [95] }\end{array}$ & $\begin{array}{l}\text { Saudi Arabia } \\
\text { (Majmaah City) }\end{array}$ & $\begin{array}{l}\text { Quantitative } \\
\text { Cross-sectional } \\
\text { Print-based } \\
\text { questionnaire } \\
\text { survey }\end{array}$ & $\begin{array}{l}\text { Medical } \\
\text { students }\end{array}$ & 65 & $\begin{array}{l}\text { Complementary and alternative } \\
\text { medicine }\end{array}$ & $\begin{array}{l}\text { Prior to T\&CM training } 38.5 \% \text { agreed the } \\
\text { unavailability of credentialled practitioners } \\
\text { was a barrier to their use in Western medical } \\
\text { settings, increasing to } 70.8 \% \text { following train- } \\
\text { ing }(p=0.0006)\end{array}$ \\
\hline $\begin{array}{l}\text { Barnes } 2018 \\
\quad[61]\end{array}$ & New Zealand & $\begin{array}{l}\text { Qualitative } \\
\text { Semi-structured } \\
\text { telephone inter- } \\
\text { views }\end{array}$ & $\begin{array}{l}\text { Practising } \\
\text { community } \\
\text { pharmacists }\end{array}$ & 27 & Complementary medicine & $\begin{array}{l}\text { Regulation of practitioners such as herbalists } \\
\text { and naturopaths was important, although } \\
\text { there were mixed views about whether } \\
\text { governance should be statutory or self- } \\
\text { regulation } \\
\text { Some suggested that access to certain } \\
\text { medicinal plant preparations should only be } \\
\text { through registered CMs practitioners, such } \\
\text { as herbalists } \\
\text { Some framed the need for regulation in terms } \\
\text { of providing recognition and validation of } \\
\text { practitioner expertise }\end{array}$ \\
\hline $\begin{array}{l}\text { Cavaco } 2017 \\
\text { [72] }\end{array}$ & $\begin{array}{l}\text { Portugal (Lisbon } \\
\text { and Porto) }\end{array}$ & $\begin{array}{l}\text { Qualitative } \\
\text { Semi-structured } \\
\text { interviews }\end{array}$ & $\begin{array}{l}\text { Community } \\
\text { pharmacists }\end{array}$ & 6 & $\begin{array}{l}\text { Homeopathic dispensing by phar- } \\
\text { macists/non-pharmacists }\end{array}$ & $\begin{array}{l}\text { There were no restrictions regarding dispens- } \\
\text { ing homeopathic medicines in pharmacies } \\
\text { Participants commonly mentioned the need } \\
\text { for regulation of homeopathic practition- } \\
\text { ers, particularly in relation to dispensing by } \\
\text { non-pharmacists } \\
\text { Improved legislation would benefit prescrib- } \\
\text { ers, increase legal protection, and improve } \\
\text { social recognition }\end{array}$ \\
\hline $\begin{array}{l}\text { Chaterji } 2007 \\
\text { [81] }\end{array}$ & $\begin{array}{l}\text { USA (Washing- } \\
\text { ton) }\end{array}$ & $\begin{array}{l}\text { Quantitative Print- } \\
\text { based question- } \\
\text { naire survey }\end{array}$ & $\begin{array}{l}\text { Medical } \\
\text { students }\end{array}$ & 266 & $\begin{array}{l}\text { Acupuncture, aromatherapy, } \\
\text { biofeedback, chiropractic, } \\
\text { herbal medicine, homeopathy, } \\
\text { hypnosis/guided imagery, } \\
\text { magnets, massage, meditation, } \\
\text { music, nutritional supplements, } \\
\text { prayer/spiritual healing, rolfing, } \\
\text { therapeutic touch }\end{array}$ & $\begin{array}{l}71.8 \% \text { agreed that the lack of credentialled } \\
\text { providers was a barrier to the use of prac- } \\
\text { tices in Western medical settings }\end{array}$ \\
\hline
\end{tabular}


Table 4 (continued)

\begin{tabular}{|c|c|c|c|c|c|}
\hline $\begin{array}{l}\text { First author } \\
\text { year }\end{array}$ & $\begin{array}{l}\text { Country } \\
\text { (jurisdiction, if } \\
\text { limited) }\end{array}$ & Study design & $\begin{array}{l}\text { Stakeholder } \\
\text { population }\end{array}$ & Sample N & T\&CM profession/s examined \\
\hline $\begin{array}{l}\text { Cohen } 2005 \\
\text { [101] }\end{array}$ & Australia & $\begin{array}{l}\text { Quantitative. } \\
\text { National postal } \\
\text { questionnaire } \\
\text { survey }\end{array}$ & $\begin{array}{l}\text { General practi- } \\
\text { tioners }\end{array}$ & 579 & $\begin{array}{l}\text { Acupuncture, aromatherapy, } \\
\text { Chinese herbal medicine, } \\
\text { chiropractic, herbal medicine, } \\
\text { homeopathy, hypnosis, mas- } \\
\text { sage, meditation, naturopathy, } \\
\text { osteopathy, reflexology, spiritual } \\
\text { healing (e.g. reiki), vitamin and } \\
\text { mineral therapy, yoga }\end{array}$ \\
\hline
\end{tabular}

Main findings

The practices that required regulation were chiropractic (88\% agreed), acupuncture

(87\%), Chinese herbal medicine (80\%), hypnosis (79\%), herbal medicine (77\%), naturopathy (73\%), osteopathy (72\%), homeopathy (66\%), vitamin and mineral therapy (66\%), yoga (49\%), meditation (44\%), spiritual healing (e.g. reiki) (36\%), aromatherapy (34\%), massage (33\%), and reflexology (28\%)

The practices that government should not regulate were: yoga (49\% agreed), meditation (44\%), spiritual healing eg. reiki (36\%), aromatherapy (34\%), massage (33\%), reflexology (28\%), vitamin and mineral therapy (18\%), homeopathy (13\%), naturopathy $(11 \%)$, osteopathy $(10 \%)$, hypnosis (9\%), herbal medicine (8\%), Chinese herbal medicine (7\%), acupuncture (6\%), and chiropractic (4\%)

Areas of greatest concern were incorrect/ inadequate/delayed diagnosis, interactions between complementary medicines and pharmaceuticals, and patients not disclosing T\&CM use to their doctors

Some commented that complementary therapies caused no harm in the hands of appropriately trained practitioners

Many GPs indicated that therapies that did not involve medicines (such as yoga, meditation, and spiritual healing) did not require regulation, whereas others that had potential to delay diagnosis or interact with conventional medication (such as herbal medicine or naturopathy) should be regulated

Some suggested self-regulation (e.g. industry accreditation) was more appropriate for some therapies than government regulation

\begin{tabular}{|c|c|c|c|c|c|}
\hline $\begin{array}{l}\text { Dooley } 2010 \\
\text { [75] }\end{array}$ & $\begin{array}{l}\text { Australia } \\
\text { (Gold Coast, } \\
\text { Melbourne, } \\
\text { and Wagga } \\
\text { Wagga) }\end{array}$ & $\begin{array}{l}\text { Quantitative. Postal, } \\
\text { online, and print- } \\
\text { based question- } \\
\text { naire survey }\end{array}$ & $\begin{array}{l}\text { Pharmacy } \\
\text { assistants }\end{array}$ & 107 & $\begin{array}{l}\text { Western herbal medicine, natur- } \\
\text { opathy }\end{array}$ \\
\hline $\begin{array}{l}\text { Flower } 2015 \\
\quad[62]\end{array}$ & UK & $\begin{array}{l}\text { Qualitative } \\
\text { Semi-structured } \\
\text { face to face } \\
\text { and telephone } \\
\text { interviews }\end{array}$ & $\begin{array}{l}\text { General practi- } \\
\text { tioners }\end{array}$ & 15 & Herbal medicine \\
\hline
\end{tabular}

$75 \%$ strongly agreed/agreed naturopaths should be formally registered to safeguard the public, 2\% disagreed, $22 \%$ were unsure

Practitioner regulation and proper quality control procedures were key factors that were required before herbal treatments could be recommended

Many were open to the use of herbal medicines when conventional treatment had failed

Concerns were expressed about the lack of quality assurance of herbal products, potential adulteration with pharmaceuticals, and possible interactions between herbs and drugs

The training, lack of regulation or licensing, and the level of medical knowledge of herbal practitioners, were additional sources of uncertainty 
Table 4 (continued)

\begin{tabular}{|c|c|c|c|c|c|c|}
\hline $\begin{array}{l}\text { First author } \\
\text { year }\end{array}$ & $\begin{array}{l}\text { Country } \\
\text { (jurisdiction, if } \\
\text { limited) }\end{array}$ & Study design & $\begin{array}{l}\text { Stakeholder } \\
\text { population }\end{array}$ & Sample N & T\&CM profession/s examined & Main findings \\
\hline Hall 2000 [103] & Australia (Perth) & $\begin{array}{l}\text { Quantitative Cross- } \\
\text { sectional postal } \\
\text { questionnaire } \\
\text { survey }\end{array}$ & $\begin{array}{l}\text { General practi- } \\
\text { tioners }\end{array}$ & 282 & $\begin{array}{l}\text { Acupuncture, aromatherapy, } \\
\text { herbal medicine, homeopathy, } \\
\text { hypnosis, massage, naturopathy, } \\
\text { meditation, spinal manipula- } \\
\text { tion, yoga }\end{array}$ & $\begin{array}{l}\text { 32.2\% did not favour referral to practitioners } \\
\text { citing lack of government regulation and } \\
\text { training standards } \\
\text { GPs were most likely to refer patients to } \\
\text { acupuncture ( } 75.0 \% \text { of respondents), mas- } \\
\text { sage }(62.0 \%) \text {, meditation ( } 53.0 \%) \text {, and yoga } \\
\text { ( } 42.0 \%) \\
\text { Fewer than } 8 \% \text { would refer patients to aroma- } \\
\text { therapy, herbal medicine, homeopathy, or } \\
\text { naturopathy }\end{array}$ \\
\hline Harris 2006 [84] & USA (Minnesota) & $\begin{array}{l}\text { Quantitative Print- } \\
\text { based question- } \\
\text { naire survey }\end{array}$ & $\begin{array}{l}\text { College of } \\
\text { Pharmacy } \\
\text { faculty } \\
\text { department } \\
\text { mem- } \\
\text { bers, and } \\
\text { pharmacy } \\
\text { students }\end{array}$ & 94 & $\begin{array}{l}\text { Acupuncture, aromatherapy, } \\
\text { bioelectromagnetic therapies, } \\
\text { biofeedback, chiropractic, } \\
\text { herbal medicine, homeopathy, } \\
\text { hypnosis/guided imagery, mas- } \\
\text { sage, music, nutritional supple- } \\
\text { ments, prayer/spiritual healing, } \\
\text { meditation, rolfing, therapeutic/ } \\
\text { healing touch }\end{array}$ & $\begin{array}{l}58 \% \text { of faculty members and } 80 \% \text { of students } \\
\text { ( } p=0.074 \text { ) agreed the unavailability of } \\
\text { credentialled providers was a barrier to the } \\
\text { use of T\&CM practices } \\
74 \% \text { of faculty members and } 53 \% \text { of students } \\
\text { indicated chiropractic a mainstream health } \\
\text { care practice } \\
53 \% \text { of students indicated nutritional supple- } \\
\text { ments mainstream practice } \\
\text { No other practice achieved }>50 \% \text { agreement } \\
\text { on being a mainstream practice }\end{array}$ \\
\hline $\begin{array}{l}\text { James } 2020 \\
\quad[59]\end{array}$ & $\begin{array}{l}\text { Sierra Leone } \\
\text { (Freetown in } \\
\text { Western Area, } \\
\text { Bo district } \\
\text { in Southern } \\
\text { Region, Ken- } \\
\text { ema district } \\
\text { in Eastern } \\
\text { Region, Bom- } \\
\text { bali district } \\
\text { in Northern } \\
\text { Region) }\end{array}$ & $\begin{array}{l}\text { Qualitative } \\
\text { Semi-structured } \\
\text { interviews }\end{array}$ & $\begin{array}{l}\text { Doctors, } \\
\text { nurses, and } \\
\text { community } \\
\text { health } \\
\text { officers }\end{array}$ & 15 & $\begin{array}{l}\text { Traditional and complementary } \\
\text { medicine }\end{array}$ & $\begin{array}{l}\text { The prerequisites for health care integration } \\
\text { included training of T\&CM practitioners on } \\
\text { what diseases to treat and what to refer, } \\
\text { regulation of practices, and public educa- } \\
\text { tion to seek care from licensed practitioners } \\
\text { only } \\
\text { T\&CM practitioners should be adequately } \\
\text { educated in basic medical training } \\
\text { Collaboration with some T\&CM practices, such } \\
\text { as herbal medicine, which were perceived } \\
\text { to cause serious adverse effects, should not } \\
\text { be permitted }\end{array}$ \\
\hline Jarvis 2015 [61] & $\begin{array}{l}\text { England } \\
\text { (Liverpool and } \\
\text { Manchester) }\end{array}$ & $\begin{array}{l}\text { Qualitative } \\
\text { Semi-structured } \\
\text { telephone inter- } \\
\text { views }\end{array}$ & $\begin{array}{l}\text { General practi- } \\
\text { tioners }\end{array}$ & 19 & $\begin{array}{l}\text { Complementary and alternative } \\
\text { medicine }\end{array}$ & $\begin{array}{l}\text { Practitioners should be professionally } \\
\text { regulated } \\
\text { GPs were significant concerned about herbal } \\
\text { remedies (e.g. causing interactions with } \\
\text { pharmaceuticals) } \\
\text { Having greater confidence in the robustness } \\
\text { of practitioner training and regulatory } \\
\text { procedures enables GPs to have greater } \\
\text { confidence in endorsing practices and refer- } \\
\text { ring patients to practitioners }\end{array}$ \\
\hline $\begin{array}{l}\text { Langworthy }^{b} \\
2000[85]\end{array}$ & Netherlands & $\begin{array}{l}\text { Quantitative Postal } \\
\text { questionnaire } \\
\text { survey }\end{array}$ & $\begin{array}{l}\text { Osteopaths, } \\
\text { manual } \\
\text { therapists, } \\
\text { physiothera- } \\
\text { pists }\end{array}$ & 227 & Chiropractic & $\begin{array}{l}59 \% \text { of osteopaths ( } n=49), 24 \% \text { of manual } \\
\text { therapists }(n=46) \text {, and } 15 \% \text { of physiothera- } \\
\text { pists ( } n=132 \text { ) agreed chiropractors should } \\
\text { be 'politically recognized'through statutory } \\
\text { registration } \\
13 \% \text { of manual therapists and } 3 \% \text { of physi- } \\
\text { otherapists agreed chiropractors should not } \\
\text { be 'politically recognized'through statutory } \\
\text { registration } \\
67 \% \text { of manual therapists agreed chiropractic } \\
\text { was in competition with manual therapy } \\
22 \% \text { of osteopaths agreed chiropractic was in } \\
\text { competition with osteopathy } \\
19 \% \text { of physiotherapists agreed chiropractic } \\
\text { was in competition with physiotherapy }\end{array}$ \\
\hline $\begin{array}{l}\text { Livingston } 2010 \\
\quad[86]\end{array}$ & $\begin{array}{l}\text { Australia (Syd- } \\
\text { ney) }\end{array}$ & $\begin{array}{l}\text { Quantitative Postal } \\
\text { questionnaire } \\
\text { survey }\end{array}$ & $\begin{array}{l}\text { General practi- } \\
\text { tioners }\end{array}$ & 288 & Herbal medicine, naturopathy & $\begin{array}{l}\text { 91\% strongly supported a national register for } \\
\text { naturopaths and herbalists, requiring qualifi- } \\
\text { cations for listed members to be licensed } \\
84.0 \% \text { agreed that herbal therapies should be } \\
\text { regulated in the same way as pharmaceuti- } \\
\text { cals, } 7.3 \% \text { disagreed }\end{array}$ \\
\hline
\end{tabular}


Table 4 (continued)

\begin{tabular}{|c|c|c|c|c|c|c|}
\hline $\begin{array}{l}\text { First author } \\
\text { year }\end{array}$ & $\begin{array}{l}\text { Country } \\
\text { (jurisdiction, if } \\
\text { limited) }\end{array}$ & Study design & $\begin{array}{l}\text { Stakeholder } \\
\text { population }\end{array}$ & Sample N & T\&CM profession/s examined & Main findings \\
\hline $\begin{array}{c}\text { Montbriand } \\
2000 \text { [87] }\end{array}$ & $\begin{array}{l}\text { Canada (Sas- } \\
\text { katchewan) }\end{array}$ & $\begin{array}{l}\text { Quantitative Postal } \\
\text { questionnaire } \\
\text { survey }\end{array}$ & $\begin{array}{l}\text { Nurses, } \\
\text { pharmacists, } \\
\text { physicians }\end{array}$ & 153 & Alternative therapies & $\begin{array}{l}\text { Across all three professional groups } 69 \% \\
\text { agreed with regulation, } 10 \% \text { did not, } 21 \% \\
\text { were undecided } \\
82 \% \text { of pharmacists ( } n=49 \text { ) agreed T\&CM } \\
\text { should be regulated or have government } \\
\text { controls, } 10 \% \text { did not agree, } 8 \% \text { did not } \\
\text { know } \\
\text { Pharmacists were alarmed at the number of } \\
\text { herbal and homeopathic products entering } \\
\text { the market without standards, regulations } \\
\text { and proper labelling } \\
69 \% \text { of physicians ( } n=52 \text { ) agreed T\&CM } \\
\text { should be regulated or have government } \\
\text { controls, } 15 \% \text { did not agree, } 15 \% \text { did not } \\
\text { know } \\
\text { Physicians felt practices with potentially harm- } \\
\text { ful side-effects should be regulated, but } \\
\text { were concerned about the impracticality of } \\
\text { controls and whether effective regulation } \\
\text { was possible } \\
56 \% \text { of nurses ( } n=52 \text { ) agreed T\&CM should } \\
\text { be regulated or have government controls, } \\
6 \% \text { did not agree, } 38 \% \text { did not know } \\
\text { Nurses focussed comments on practitioners, } \\
\text { expressing the need for self- or government } \\
\text { regulation and certification to practice }\end{array}$ \\
\hline Morin 2017 [88] & $\begin{array}{l}\text { Canada (Que- } \\
\text { bec) }\end{array}$ & $\begin{array}{l}\text { Quantitative Postal } \\
\text { questionnaire } \\
\text { survey }\end{array}$ & Physicians & 266 & Osteopathy & $\begin{array}{l}\text { Physicians supported the regulation of } \\
\text { osteopathy and indicated it would promote } \\
\text { collaboration } \\
72 \% \text { would be greatly/moderately influenced } \\
\text { by government regulation and the estab- } \\
\text { lishment of university-based programs, } 28 \% \\
\text { would not be influenced/very influenced } \\
\text { by regulation } \\
\text { Physicians indicated regulation would } \\
\text { promote collaboration, } 85.3 \% \text { agreeing that } \\
\text { collaborating with osteopaths was slightly/ } \\
\text { quite/very important }\end{array}$ \\
\hline $\begin{array}{l}\text { Poreddi } 2016 \\
\quad[104]\end{array}$ & India (Bangalore) & $\begin{array}{l}\text { Quantitative } \\
\text { Cross-sectional } \\
\text { Print-based } \\
\text { questionnaire } \\
\text { survey }\end{array}$ & Student nurses & 122 & $\begin{array}{l}\text { Acupuncture, Ayurveda, } \\
\text { aromatherapy, biofeedback, } \\
\text { chiropractic, herbal medicine, } \\
\text { homeopathy, hypnosis, natur- } \\
\text { opathy, reflexology, spiritual } \\
\text { healing }\end{array}$ & $\begin{array}{l}\text { 81.1\% agreed that the unavailability of creden- } \\
\text { tialled providers was a barrier to the use of } \\
\text { T\&CM, } 18.9 \% \text { did not agree }\end{array}$ \\
\hline $\begin{array}{l}\text { Poynton } 2006 \\
\text { [98] }\end{array}$ & New Zealand & $\begin{array}{l}\text { Quantitative. } \\
\text { Nationwide cross- } \\
\text { sectional postal } \\
\text { questionnaire } \\
\text { survey }\end{array}$ & $\begin{array}{l}\text { General practi- } \\
\text { tioners }\end{array}$ & 300 & $\begin{array}{l}\text { Acupuncture, aromatherapy, } \\
\text { traditional Chinese medicine, } \\
\text { chiropractic, herbal medi- } \\
\text { cine, homeopathy, hypnosis, } \\
\text { traditional Māori medicine, } \\
\text { naturopathy, osteopathy, tradi- } \\
\text { tional Pacific Island medicine, } \\
\text { reflexology, spiritual healing }\end{array}$ & $\begin{array}{l}\text { Less than } 12.3 \% \text { of GPs referred patients to } \\
\text { aromatherapy, herbal medicine, natur- } \\
\text { opathy and traditional Chinese medicine, } \\
\text { traditional Māori medicine, and traditional } \\
\text { Pacific Island medicine, compared to greater } \\
\text { than } 70.0 \% \text { referral rate to acupuncture, } \\
\text { chiropractic, and osteopathy } \\
\text { The most common reasons for not referring } \\
\text { patients were lack of evidence ( } 88 \% \text {, } \\
n=264) \text {, lack of regulation ( } 78 \%, n=234) \text {, } \\
\text { and financial cost to patients ( } 50.3 \%, \\
n=151) \\
\text { Other reasons for non-referral include } \\
\text { concerns about exploitation of vulnerable } \\
\text { patients and the risk of adverse effects or } \\
\text { harm }\end{array}$ \\
\hline
\end{tabular}


Table 4 (continued)

\begin{tabular}{|c|c|c|c|c|c|c|}
\hline $\begin{array}{l}\text { First author } \\
\text { year }\end{array}$ & $\begin{array}{l}\text { Country } \\
\text { (jurisdiction, if } \\
\text { limited) }\end{array}$ & Study design & $\begin{array}{l}\text { Stakeholder } \\
\text { population }\end{array}$ & Sample N & T\&CM profession/s examined & Main findings \\
\hline Price 2004 [89] & UK & $\begin{array}{l}\text { Quantitative. } \\
\text { Population-based } \\
\text { postal question- } \\
\text { naire survey }\end{array}$ & $\begin{array}{l}\text { Members of } \\
\text { the British } \\
\text { Medical } \\
\text { Acupuncture } \\
\text { Society } \\
\text { (including } \\
\text { general } \\
\text { practition- } \\
\text { ers (75\% of } \\
\text { respond- } \\
\text { ents), } \\
\text { hospital } \\
\text { doctors and } \\
\text { other health } \\
\text { profession- } \\
\text { als) }\end{array}$ & 1112 & Acupuncture & $\begin{array}{l}56 \% \text { favoured of some form of regulatory } \\
\text { governing body for practitioners, } 27 \% \text { were } \\
\text { undecided }\end{array}$ \\
\hline $\begin{array}{l}\text { Semple } 2006 \\
\text { [90] }\end{array}$ & $\begin{array}{l}\text { Australia (South } \\
\text { Australia, } \\
\text { Queensland, } \\
\text { and Victoria) }\end{array}$ & $\begin{array}{l}\text { Quantitative Postal } \\
\text { questionnaire } \\
\text { survey }\end{array}$ & $\begin{array}{l}\text { Practising } \\
\text { pharmacists }\end{array}$ & 211 & $\begin{array}{l}\text { Complementary and alternative } \\
\text { medicine practices dispensing } \\
\text { vitamins and minerals, flower } \\
\text { remedies, herbal products and } \\
\text { other dietary supplements, } \\
\text { homeopathic products, tissue } \\
\text { salts }\end{array}$ & $\begin{array}{l}\text { Pharmacists agreed the current level of } \\
\text { regulation of practitioners was a barrier } \\
\text { to information provision about T\&CM to } \\
\text { consumers. One way of overcoming this } \\
\text { was indicated to be better regulation of } \\
\text { practitioners }\end{array}$ \\
\hline Taylor 2003 [91] & $\begin{array}{l}\text { New Zealand } \\
\text { (Wanganui) }\end{array}$ & $\begin{array}{l}\text { Quantitative Postal } \\
\text { questionnaire } \\
\text { survey }\end{array}$ & $\begin{array}{l}\text { General practi- } \\
\text { tioners }\end{array}$ & 25 & $\begin{array}{l}\text { Acupuncture, aromatherapy, } \\
\text { chiropractic, colour therapy, } \\
\text { homeopathy, hypnosis, iridol- } \\
\text { ogy, reflexology, Rongoā Māori }\end{array}$ & $\begin{array}{l}84 \% \text { indicated they would like to see better } \\
\text { regulation of therapies } \\
96 \% \text { indicated concern about the safety of } \\
\text { therapies }\end{array}$ \\
\hline $\begin{array}{l}\text { Tiralongo } 2010 \\
\text { [99] }\end{array}$ & Australia & $\begin{array}{l}\text { Quantitative. } \\
\text { National postal } \\
\text { questionnaire } \\
\text { survey }\end{array}$ & $\begin{array}{l}\text { Community, } \\
\text { hospital, } \\
\text { consultant, } \\
\text { industry, } \\
\text { academia, } \\
\text { public } \\
\text { service, and } \\
\text { army phar- } \\
\text { macists }\end{array}$ & 583 & Herbal medicine, naturopathy & $\begin{array}{l}92 \% \text { strongly agreed/agreed that practitioners } \\
\text { should be formally registered to safeguard } \\
\text { the public, 3\% disagreed/strongly disagreed, } \\
5 \% \text { were unsure }\end{array}$ \\
\hline $\begin{array}{l}\text { Wardle } 2018 \\
\text { [51] }\end{array}$ & $\begin{array}{l}\text { Australia (Non- } \\
\text { metropolitan } \\
\text { New South } \\
\text { Wales) }\end{array}$ & $\begin{array}{l}\text { Qualitative. } \\
\text { Population-based } \\
\text { online and postal } \\
\text { questionnaire } \\
\text { survey analysis } \\
\text { of open-ended } \\
\text { question }\end{array}$ & $\begin{array}{l}\text { General practi- } \\
\text { tioners }\end{array}$ & 152 & Complementary medicine & $\begin{array}{l}\text { Risk was a major theme in many of the } \\
\text { responses, with both direct (e.g. drug-herb } \\
\text { interaction, adverse events), and indirect } \\
\text { risks (e.g. delayed diagnosis, exploitation) } \\
\text { identified } \\
\text { Most held that risks were maximised due to } \\
\text { the variability of standards, practices and } \\
\text { treatments which was related in large part } \\
\text { to the regulatory vacuum } \\
\text { Practitioner or product variability or potential } \\
\text { monopolisation of care by providers were } \\
\text { highlighted as major issues that increased } \\
\text { potential risks to patients. For many GPs, it } \\
\text { was this risk, rather than risk of ineffective } \\
\text { therapies, which was of most concern } \\
\text { Both supporters and detractors of T\&CM were } \\
\text { concerned about the lack of regulation } \\
\text { Providers practised in a regulatory vacuum } \\
\text { and were considered to be practising with- } \\
\text { out any regulatory oversight and therefore } \\
\text { without restrictions }\end{array}$ \\
\hline
\end{tabular}


Table 4 (continued)

\begin{tabular}{|c|c|c|c|c|c|c|}
\hline $\begin{array}{l}\text { First author } \\
\text { year }\end{array}$ & $\begin{array}{l}\text { Country } \\
\text { (jurisdiction, if } \\
\text { limited) }\end{array}$ & Study design & $\begin{array}{l}\text { Stakeholder } \\
\text { population }\end{array}$ & Sample N & T\&CM profession/s examined & Main findings \\
\hline$Y^{C}{ }^{c} 2015$ [100] & Korea & $\begin{array}{l}\text { Quantitative Email, } \\
\text { postal and print- } \\
\text { based question- } \\
\text { naire survey }\end{array}$ & $\begin{array}{l}\text { Doctors, } \\
\text { nurses, } \\
\text { Oriental } \\
\text { (Korean) } \\
\text { medical } \\
\text { doctors as } \\
\text { medical pro- } \\
\text { fessionals }\end{array}$ & 19 & $\begin{array}{l}\text { Acupuncture, alternative medi- } \\
\text { cine, chiropractic, clinical art, } \\
\text { feet massage, laughter, Qi gong, } \\
\text { trainees of traditional Chinese } \\
\text { medicine }\end{array}$ & $\begin{array}{l}\text { Across all provider groups, medical and T\&CM } \\
\text { professionals, } 32.8 \% \text { agreed legislation to } \\
\text { govern providers was preferable, } 25.0 \% \\
\text { agreed with qualifications/accreditation, } \\
13.6 \% \text { agreed with making/evaluating } \\
\text { standards } \\
\text { 31.6\% agreed government administered } \\
\text { examinations were the most important for } \\
\text { gaining T\&CM qualifications, } 15.8 \% \text { agreed } \\
\text { that qualifications from a certified institute, } \\
\text { e.g. college/university was preferable } \\
\text { 29.4\% agreed a national examination for man- } \\
\text { aging qualifications was preferable, } 23.6 \% \\
\text { agreed with legislation and national control } \\
\text { as the preferred option }\end{array}$ \\
\hline \multicolumn{7}{|c|}{ Professional associations $(n=6)$} \\
\hline Clarke 2004 [54] & UK & $\begin{array}{l}\text { Qualitative. Docu- } \\
\text { ment analysis }\end{array}$ & $\begin{array}{l}\text { T\&CM } \\
\text { professional } \\
\text { associations } \\
\text { representing } \\
\text { aromather- } \\
\text { apy, Chinese } \\
\text { herbal } \\
\text { medicine, } \\
\text { chiroprac- } \\
\text { tic, crystal } \\
\text { healing, feng } \\
\text { shui, 'lay' } \\
\text { homeopathy, } \\
\text { medical } \\
\text { homeopathy, } \\
\text { osteopathy } \\
\text { and radionics }\end{array}$ & 9 & $\begin{array}{l}\text { Aromatherapy, Chinese herbal } \\
\text { medicine, chiropractic, crystal } \\
\text { healing, feng shui, 'lay' home- } \\
\text { opathy, medical homeopathy, } \\
\text { osteopathy, radionics }\end{array}$ & $\begin{array}{l}\text { Most associations promoted the need for } \\
\text { tighter regulation } \\
\text { Professionalisation strategies were necessary } \\
\text { to eliminate unprofessional conduct, ensure } \\
\text { autonomy of practice and promote legiti- } \\
\text { macy of practitioners } \\
\text { The Chinese herbalists associations and chiro- } \\
\text { practic associations were most concerned } \\
\text { with professionalisation } \\
\text { The Chinese herbalists associations were com- } \\
\text { mitted to statutory self-regulation arguing } \\
\text { this was the best option to ensure profes- } \\
\text { sional recognition, establish protection of } \\
\text { title and give authority to use scheduled } \\
\text { herbs } \\
\text { Leaving regulation unaddressed would risked } \\
\text { imposition of government controls } \\
\text { Regulation provided greater protection for } \\
\text { patients, increased credibility, raised the } \\
\text { visibility of herbal medicine and protected } \\
\text { practice autonomy } \\
\text { Challenges to regulation was gaining consen- } \\
\text { sus between associations } \\
\text { Concerns included dilution of philosophical } \\
\text { traditions and practice standardisation }\end{array}$ \\
\hline $\begin{array}{l}\text { Gilmour } 2002 \\
\text { [63] }\end{array}$ & Canada (Ontario) & $\begin{array}{l}\text { Qualitative. } \\
\text { Unstructured } \\
\text { interviews }\end{array}$ & $\begin{array}{l}\text { Leaders in the } \\
\text { professions } \\
\text { of acu- } \\
\text { puncture/ } \\
\text { traditional } \\
\text { Chinese } \\
\text { medicine, } \\
\text { homeopathy, } \\
\text { and natur- } \\
\text { opathy }\end{array}$ & 24 & $\begin{array}{l}\text { Acupuncture/traditional Chinese } \\
\text { medicine, homeopathy, } \\
\text { naturopathy }\end{array}$ & $\begin{array}{l}\text { Statutory self-regulation was seen as key to } \\
\text { full professionalisation by all groups. They } \\
\text { desired the protection of statutory regula- } \\
\text { tion which included status and legitimacy, } \\
\text { acknowledgement of skills and qualifica- } \\
\text { tions, potential integration into the health } \\
\text { care system, restricted use of designated } \\
\text { titles to registered members, acceptance } \\
\text { of practices by private insurers, provision } \\
\text { of a defined scope of practice, to assure } \\
\text { consumers of the quality of training and } \\
\text { protection against those who did not meet } \\
\text { the standards } \\
\text { All groups expressed the hope that regula- } \\
\text { tion would lead to the establishment and } \\
\text { enforcement of standards of practice but } \\
\text { were unable to accomplish this due to inter- } \\
\text { nal disagreements, division, lack of cohe- } \\
\text { sion, and intra-professional competition, all } \\
\text { of which hindered regulation attempts } \\
\text { Only the naturopaths saw the need for appro- } \\
\text { priate research to support the push for } \\
\text { regulation, the two remaining groups were } \\
\text { content to rely on historical evidence }\end{array}$ \\
\hline
\end{tabular}


Table 4 (continued)

\begin{tabular}{|c|c|c|c|c|c|c|}
\hline $\begin{array}{l}\text { First author } \\
\text { year }\end{array}$ & $\begin{array}{l}\text { Country } \\
\text { (jurisdiction, if } \\
\text { limited) }\end{array}$ & Study design & $\begin{array}{l}\text { Stakeholder } \\
\text { population }\end{array}$ & Sample N & T\&CM profession/s examined & Main findings \\
\hline $\begin{array}{l}\text { Kelner } 2004 \\
\text { [67] }\end{array}$ & Canada (Ontario) & $\begin{array}{l}\text { Qualitative } \\
\text { Semi-structured } \\
\text { interviews, and } \\
\text { published policy } \\
\text { material }\end{array}$ & $\begin{array}{l}\text { Directors or } \\
\text { presidents of } \\
\text { conventional } \\
\text { professional } \\
\text { associations } \\
\text { of medicine, } \\
\text { nursing, } \\
\text { physiother- } \\
\text { apy, clinical } \\
\text { nutrition } \\
\text { and public } \\
\text { health, and } \\
\text { federal/ } \\
\text { provincial } \\
\text { statutory } \\
\text { governing } \\
\text { bodies of the } \\
\text { professions } \\
\text { of clinical } \\
\text { nutrition, } \\
\text { medicine, } \\
\text { nursing, } \\
\text { physi- } \\
\text { otherapy, } \\
\text { and public } \\
\text { health }\end{array}$ & 10 & $\begin{array}{l}\text { Acupuncture/traditional Chinese } \\
\text { medicine, chiropractic, home- } \\
\text { opathy, naturopathy, reiki }\end{array}$ & $\begin{array}{l}\text { Most were unsympathetic to the professionali- } \\
\text { sation of T\&CM groups } \\
\text { Achieving statutory self-regulation was impor- } \\
\text { tant for professionalisation but had to be } \\
\text { earned. There was a reluctance to encour- } \\
\text { age T\&CM groups to gain regulation } \\
\text { They argued that unless therapies had a body } \\
\text { of knowledge based on scientific evidence } \\
\text { and a method of delivering care in an objec- } \\
\text { tive, standardised way, it was unsafe to allow } \\
\text { practitioners to treat patients } \\
\text { Higher standards of evidence were essential } \\
\text { to gain formal recognition and a place in } \\
\text { the health care system } \\
\text { In order for T\&CM practitioners to be creden- } \\
\text { tialled, their therapies and practices would } \\
\text { need to be evidence-based } \\
\text { Nursing representatives more often empha- } \\
\text { sised the need for public safety and protec- } \\
\text { tion as a rationale for regulating T\&CM } \\
\text { providers, rather than a need for evidence } \\
\text { Allied health professions felt strongly that } \\
\text { regulation should not be granted until there } \\
\text { was scientific evidence that therapies were } \\
\text { safe and effective and scopes of practice } \\
\text { were suitable } \\
\text { Regulation would promote referrals, especially } \\
\text { from the nursing profession } \\
\text { The issue of scope of practice evoked tensions } \\
\text { and concerns about maintaining jurisdic- } \\
\text { tional boundaries and protecting turf } \\
\text { Leaders proposed several ways to block or } \\
\text { control integration; co-optation, physicians } \\
\text { as gatekeepers, and opposing government } \\
\text { funding for T\&CM }\end{array}$ \\
\hline $\begin{array}{l}\text { Kelner } 2006 \\
\text { [68] }\end{array}$ & Canada (Ontario) & $\begin{array}{l}\text { Qualitative } \\
\text { Semi-structured } \\
\text { interviews, and } \\
\text { documentary } \\
\text { material }\end{array}$ & $\begin{array}{l}\text { Leaders in the } \\
\text { professions } \\
\text { of chiro- } \\
\text { practic and } \\
\text { homeopathy }\end{array}$ & 16 & Chiropractic, homeopathy & $\begin{array}{l}\text { Common professionalisation strategies were } \\
\text { used by both professions, i.e. improving } \\
\text { education quality, raising practice standards, } \\
\text { developing research capacity, and increas- } \\
\text { ing group cohesion } \\
\text { There was insufficient support from govern- } \\
\text { ment for professionalisation efforts } \\
\text { Achieving statutory self-regulation motivated } \\
\text { chiropractors to pursue professionalisation } \\
\text { strategies } \\
\text { Regulation gave authority to enforce practice } \\
\text { standards and monitor ethical misconduct, } \\
\text { but did not improve professional harmony } \\
\text { As an unregulated profession, homeopathy } \\
\text { had scarce resources, and greater difficulty } \\
\text { in maintaining clinical standards and } \\
\text { sanctioning unethical practitioners because } \\
\text { of inadequate monitoring. They also had } \\
\text { greater internal division which impeded } \\
\text { professionalisation efforts } \\
\text { Some homeopathy leaders believed regula- } \\
\text { tion would promote cohesion and raise } \\
\text { practice standards }\end{array}$ \\
\hline
\end{tabular}


Table 4 (continued)

\begin{tabular}{|c|c|c|c|c|c|}
\hline $\begin{array}{l}\text { First author } \\
\text { year }\end{array}$ & $\begin{array}{l}\text { Country } \\
\text { (jurisdiction, if } \\
\text { limited) }\end{array}$ & Study design & $\begin{array}{l}\text { Stakeholder } \\
\text { population }\end{array}$ & Sample N & T\&CM profession/s examined \\
\hline $\begin{array}{l}\text { Lin } 2005 \text { (Sec- } \\
\text { tion 6, McCabe) } \\
\text { [52] (Summa- } \\
\text { rised in [105]) }\end{array}$ & Australia & $\begin{array}{l}\text { Quantitative Postal } \\
\text { questionnaire } \\
\text { survey }\end{array}$ & $\begin{array}{l}\text { T\&CM profes- } \\
\text { sional asso- } \\
\text { ciations of } \\
\text { naturopathy } \\
\text { and Western } \\
\text { herbal } \\
\text { medicine }\end{array}$ & 11 & $\begin{array}{l}\text { Western herbal medicine, natur- } \\
\text { opathy }\end{array}$ \\
\hline
\end{tabular}

Main findings

Welsh $^{\mathrm{d}} 2004$

[70]
Canada (Ontario)

Qualitative

Semi-structured
interviews
T\&CM senior

leaders

of the

associations

of traditional

Chinese

medicine/

acupuncture,

homeopathy,

and natur-

opathy
$36 \%$ of associations supported statutory regulation, $36 \%$ supported the existing self-regulatory model, $27 \%$ wanted stronger regulation (unspecified)

$64 \%$ of associations were negative about the existing self-regulatory model, in part because it had not produced a national, consistent, or effective regulatory system

The need to protect the professions and practices was seen as strong motivators by supporters and opposers of statutory regulation

There was no uniform minimum standard of education, and concerns about inadequately trained or incompetent practitioners

Some supporters of statutory regulation argued that this status, and with it raised standard of education, was essential to gain access to currently scheduled natural medicines

Others believed statutory regulation could introduce unacceptable restrictions on practice, and care should be taken that the regulatory effect on education, particularly increasing medicalisation of naturopathy, did not diminish the founding philosophies

There was potential for conflict of interest if professional associations were linked with private education providers, whose commercial interests might not be served by the requirement to raise standards of education

Strategies employed to achieve statutory self-regulation were improving education and practice standards, engaging in peerreviewed research, and increasing group cohesion

The inclusion of medical science was considered the basis of distinguishing between 'science' and 'non-science' and who should practise and who should not. All groups attempted to demarcate knowledge claims from competitors, and they all engaged in boundary work. The diversity of knowledge claims made uniform standards difficult to achieve

Leaders looked forward to the raised minimum education standards that regulation would impose but differing educational standards between homeopathy schools impeded the setting of uniform standards. High standards were seen as important to protect the public

Some saw the need for more clinical and peer-reviewed research to support regulation claims but debated what research was required

Challenges included no intra-professional agreement on the best standards to follow, which knowledges to codify or what kind of research should be conducted

Some considered encouraging more cohesion through conflict resolution to successfully deal with government and achieve regulatory status 
Table 4 (continued)

\begin{tabular}{|c|c|c|c|c|c|c|}
\hline $\begin{array}{l}\text { First author } \\
\text { year }\end{array}$ & $\begin{array}{l}\text { Country } \\
\text { (jurisdiction, if } \\
\text { limited) }\end{array}$ & Study design & $\begin{array}{l}\text { Stakeholder } \\
\text { population }\end{array}$ & Sample N & T\&CM profession/s examined & Main findings \\
\hline
\end{tabular}

\section{Education providers $(n=2)$}

Lin 2005 (Sec- Australia

tion 5, McCabe)

[52] (Summa-

rised in [80])

$\begin{array}{lll}\begin{array}{l}\text { Quantitative Postal } \\ \text { questionnaire }\end{array} & \begin{array}{l}\text { Education } \\ \text { providers of }\end{array} & \begin{array}{c}\text { Western herbal medicine, natur- } \\ \text { opathy }\end{array} \\ & \begin{array}{l}\text { undergradu- } \\ \text { ate and post- } \\ \text { graduate } \\ \text { courses }\end{array}\end{array}$

courses

\begin{tabular}{|c|c|c|c|c|c|}
\hline $\begin{array}{l}\text { Welsh }^{\mathrm{d}} 2004 \\
\quad[70]\end{array}$ & Canada (Ontario) & $\begin{array}{l}\text { Qualitative } \\
\text { Semi-structured } \\
\text { interviews }\end{array}$ & $\begin{array}{l}\text { T\&CM senior } \\
\text { leaders of } \\
\text { the major } \\
\text { schools of } \\
\text { traditional } \\
\text { Chinese } \\
\text { medicine/ } \\
\text { acupuncture, } \\
\text { homeopathy, } \\
\text { and natur- } \\
\text { opathy }\end{array}$ & 24 & $\begin{array}{l}\text { Acupuncture/traditional Chinese } \\
\text { medicine, homeopathy, } \\
\text { naturopathy }\end{array}$ \\
\hline
\end{tabular}

Overall 53\% preferred statutory regulation of practitioners

$64 \%$ of private providers $(n=14)$ supported government involvement in regulation, $36 \%$ supported statutory regulation, $28.5 \%$ supported co-regulation, $7 \%$ supported continued self-regulation

$100 \%$ of universities ( $n=5$ ) supported statutory regulation

A degree level of education was essential 'for the good of the

profession', and to provide university pathways, though only $45 \%$ of providers supported a bachelor's degree as the minimum requirement

A major concern of private providers was that moving courses into the university sector might result in a loss of traditional holistic philosophies and perspectives

There was about ongoing conflict within the professions over regulation and education

Major concerns were lack of agreement on the most appropriate model of regulation and minimum educational standards, the commercialisation of education resulting in lowered standards, lack of democratic processes and transparent policies in some professional associations, and the need for standards to be set by an independent body

Strategies employed to achieve statutory self-regulation were improving education and practice standards, engaging in peerreviewed research, and increasing group cohesion

The inclusion of medical science was considered the basis of distinguishing between 'science' and 'non-science' and who should practise and who should not. All groups attempted to demarcate knowledge claims from competitors, and they all engaged in boundary work. The diversity of knowledge claims made uniform standards difficult to achieve

Leaders looked forward to the raised minimum education standards that regulation would impose but differing educational standards between homeopathy schools impeded the setting of uniform standards. High standards were seen as important to protect the public

Some saw the need for more clinical and peer-reviewed research to support regulation claims but debated what research was required

Challenges included no intra-professional agreement on the best standards to follow, which knowledges to codify or what kind of research should be conducted

Some considered encouraging more cohesion through conflict resolution to successfully deal with government and achieve regulatory status 
Table 4 (continued)

\begin{tabular}{|c|c|c|c|c|c|c|}
\hline $\begin{array}{l}\text { First author } \\
\text { year }\end{array}$ & $\begin{array}{l}\text { Country } \\
\text { (jurisdiction, if } \\
\text { limited) }\end{array}$ & Study design & $\begin{array}{l}\text { Stakeholder } \\
\text { population }\end{array}$ & Sample N & T\&CM profession/s examined & Main findings \\
\hline
\end{tabular}

Policy-makers $(n=2)$

Kelly 2005 [65] Canada (Alberta British Columbia)
Qualitative

Semi-structured

interviews
Regional health 10

policy-

makers
Acupuncture, chiropractic, herbal medicine, homeopathy, hypnosis, massage
Policy-makers held a positive view for the integration of T\&CM at the clinical and primary care levels of practice

Public safety was an important concern Policy-makers supported the movement towards integrative health services, but emphasised that the issues of evidencebased T\&CM research, standards of accreditation and training for T\&CM practitioners, as well as the issue of who pays, needed to be addressed to ensure the improved health and well-being of the public

CAM credibility and the potential for integration suffered from a lack of evidence demonstrating beneficial outcomes More rigorous training and licensing of T\&CM practitioners would contribute positively to movement towards integration

Poor and uneven accreditation of T\&CM practitioners was a significant barrier More thorough and consistent accreditation procedures for T\&CM practitioners would increase legitimacy in the eyes of the conventional healthcare system, the government and the general public

Some identified the need for medical training in the T\&CM curricula and viewed this step as the 'point of real leverage' for T\&CM integration

A few felt that integration was hampered by potential boundary disputes between conventional and T\&CM practitioners 
Table 4 (continued)

\begin{tabular}{|c|c|c|c|c|c|c|}
\hline $\begin{array}{l}\text { First author } \\
\text { year }\end{array}$ & $\begin{array}{l}\text { Country } \\
\text { (jurisdiction, if } \\
\text { limited) }\end{array}$ & Study design & $\begin{array}{l}\text { Stakeholder } \\
\text { population }\end{array}$ & Sample N & T\&CM profession/s examined & Main findings \\
\hline $\begin{array}{l}\text { Kelner } 2004 \\
\text { [66] }\end{array}$ & Canada (Ontario) & $\begin{array}{l}\text { Qualitative } \\
\text { Semi-structured } \\
\text { interviews }\end{array}$ & $\begin{array}{l}\text { Federal, pro- } \\
\text { vincial, and } \\
\text { municipal } \\
\text { govern- } \\
\text { ment health } \\
\text { department } \\
\text { officials }\end{array}$ & 10 & $\begin{array}{l}\text { Acupuncture/traditional Chinese } \\
\text { medicine, chiropractic, home- } \\
\text { opathy, naturopathy, reiki }\end{array}$ & 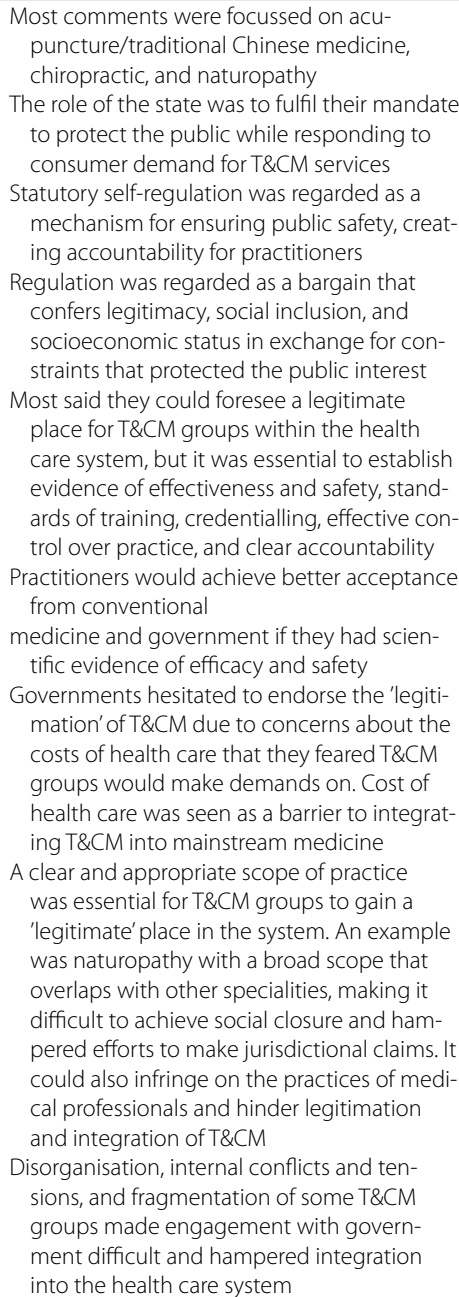 \\
\hline
\end{tabular}

a This percentage has been calculated by the first author using raw data supplied in the published article

b Physiotherapists, manual therapists and osteopaths were considered conventional medical practitioners in the country of research

c Oriental medical doctors were considered conventional medicine professionals in the country of research

d This study examined both professional association and education provider stakeholders

associations supported regulation, 36\% preferred selfregulation, and $27 \%$ called for a stronger, unspecified model of regulation [52]. Canadian T\&CM associations $[63,68,70]$ and education providers [70] reported regulation was necessary for the professionalisation of T\&CM practices. In contrast, only $53 \%$ of Australian education providers supported regulation, with significant differences between private (36\% support) and university (100\% support) sector providers [52]. Representatives of Canadian conventional medicine associations indicated reluctance for encouraging $\mathrm{T} \& \mathrm{CM}$ groups to pursue regulation and reported that while regulation may be important for $\mathrm{T} \& \mathrm{CM}$ it had to be earned through established evidence and standards [67]. Canadian policy-makers were generally supportive of $\mathrm{T} \& \mathrm{CM}$ professional regulation and integration into mainstream health provision $[65,66]$.

Consumers favoured regulation for certain $\mathrm{T} \& \mathrm{CM}$ professions, indicating it should be the same as for medical practitioners in half of all consumer studies [79, 83, 91, 93]. In decreasing order of highest reported percentage consumers in Australia, New Zealand, and UK supported the regulation of Ayurveda, naturopathy (90\%) [79], herbal medicine (90\%) 
[76, 79], homeopathy, osteopathy, reflexology (90\%) [76], Chinese medicine (76\%-90\%) [79, 93, 94], acupuncture (71\%-90\%) [76, 91, 94], aromatherapy, chiropractic, hypnotherapy (71\%-90\%) [76, 91], and Rongoā Māori (71\%) [91].

Conventional medicine practitioners also preferred certain T\&CM professions to be regulated. Studies from Australia, Netherlands, and UK reported provider support for regulation of Western herbal medicine (77\%92\%) $[86,99,101]$, naturopathy $(73 \%-92 \%)[75,86,99$, 101], chiropractic $(15 \%-88 \%)$ [85, 101], acupuncture (56\%-87\%) [89, 101], and Chinese herbal medicine (80\%) [101]. Most notable is the low support for regulating T\&CM practices reported by Korean medical professionals (33\%) [100], and for regulating chiropractors reported by physiotherapists $(15 \%)$ and manual therapists $(24 \%)$ in the Netherlands [85]. Also noteworthy in the latter study is that $19 \%$ of physiotherapists and $67 \%$ of manual therapists agreed that chiropractic was in competition with their profession [85].

\section{Public, practitioner and practice impacts of regulation}

Thirty-three studies reported stakeholder reasons for attitudes towards regulation of T\&CM practices. These attitudinal drivers were analysed inductively and summarised into three key themes of stakeholder impact; the public, T\&CM practitioners, and T\&CM practices. Full details of these findings can be found in Table 4.

\section{Regulation and the public}

Twenty studies reported stakeholder attitudes regarding the impact of regulation on the public. Studies from Australia, Canada, New Zealand, and UK reported consumer, practitioner, and policy-maker views that regulation of T\&CM practices was needed to safeguard the public [65, $66,75,94,99,101]$ and protect patients from unqualified, incompetent or unethical T\&CM practitioners [50, 52, 60, 65, 66, 69, 71, 74, 93].

Representatives of Canadian nursing associations emphasised public safety to justify their support of T\&CM regulation, however allied health association representatives stated regulation should be deferred until $T \& C M$ practices established evidence of safety and efficacy [67]. Consumers and GPs stressed the potential for harm from $\mathrm{T} \& \mathrm{CM}$ treatments including interactions with pharmaceuticals $[51,62,83,91,98,101]$, as well as harm due to patient exploitation [51, 98], financial cost $[98,101]$, and inadequate or delayed diagnosis [51, 101]. In one study GPs reported that the risk of harm was primarily due to lack of regulation [51], while in another GPs indicated that well-trained T\&CM practitioners caused little or no patient harm [101]. Conventional medicine practitioners in Sierra Leone stated that herbal medicine posed such serious risks they would not collaborate with T\&CM practitioners regardless of regulatory status [59]. Some Australian naturopaths could not agree on whether regulation would improve complaints handling [71], while consumers identified the need for an official body to hear complaints because of doubts that self-regulated professional associations would adequately sanction errant members [52].

\section{Regulation and T\&CM practitioners}

The training and qualifications of $\mathrm{T} \& \mathrm{CM}$ practitioners came under scrutiny from all stakeholder groups in 16 studies conducted in Australia, Canada, New Zealand, Sierra Leone, and UK. Consumers [76], conventional medicine practitioners [59, 62, 64, 103], and policymakers $[65,66]$ expressed concerns regarding the training, qualifications or biomedical knowledge of $\mathrm{T} \& \mathrm{CM}$ providers. T\&CM practitioners indicated that regulation would improve training standards, qualification standards $[60,69,71,73,77]$, and practitioner competence [71, 77]. However, not all Australian and Canadian T\&CM practitioners were positive about the likelihood of raised training standards due to perceptions of unfair or unachievable qualifications, grandparenting, or language proficiency requirements [50,71].

Regulation was seen to confer intra-professional and inter-professional advantages to $\mathrm{T} \& \mathrm{CM}$ practitioners. These include enhancing status and prestige $[50,71,73$, $74,77,78]$, promoting professional acceptance and recognition $[60,69,71,73,77,78]$, granting legitimacy [63, $65,66,71]$, improving inter-professional relationships $[71,77]$, encouraging greater collaboration [88], and facilitating integration into health care systems [74, 77]. GPs perceived that training quality assurance would promote patient referral to T\&CM providers [64] or conversely did not favour patient referral due to perceived inadequate training standards [103].

T\&CM education providers and representatives of professional associations noted that improving educational standards was an integral part of achieving regulation and professionalisation [52, 63, 68, 70]. Canadian policy-makers stated that poor quality $\mathrm{T} \& \mathrm{CM}$ training and credentialling practices created barriers to achieving regulatory status $[65,66]$. They indicated that $\mathrm{T} \& \mathrm{CM}$ could have a legitimate place in health care provided they demonstrated an evidence base and established appropriate standards [66].

\section{Regulation and T\&CM practice}

The impact of regulation on practice standards, occupational title protection, and scopes of practice drew most comments from T\&CM practitioners and professional 
associations in studies from Australia, Canada, New Zealand, and UK.

T\&CM practitioners anticipated that practice, professional, or ethical standards would improve following regulation [71, 73, 74, 77], while GPs indicated that variations in T\&CM standards, practices and treatments, due primarily to lack of regulation, increased risks to patients [51]. Representatives from T\&CM professional associations and analysis of association documentation indicated that regulation and professionalisation provided the means for monitoring and mandating practice standards [68], and preventing unprofessional conduct [54]. In contrast, New Zealand massage therapists regarded benefits such as practitioner monitoring, education standardisation, and professionalisation were readily provided under both self-regulatory and statutory frameworks [68]. Similarly, some Australian naturopaths and herbalists argued that regulation was unnecessary because of membership of professional associations [71, 74] that maintained and monitored practice standards [71].

A key benefit of regulation according to some T\&CM practitioners [55] and professional association representatives $[54,63]$ was the provision of legal protection of T\&CM occupational titles. Though some T\&CM practitioners were divided on the issue [77], and others were uncertain whether regulation could resolve the problem of overlapping practice scopes [50], regulation was regarded by some T\&CM practitioners [73] and association representatives [63] as facilitating the establishment of scopes of practice or practice boundaries. For conventional medicine association representatives the prospect of a T\&CM scope of practice prompted concerns over jurisdictional boundary infringements, including limiting scope for T\&CM integration [67]. This view was shared by Canadian policy-makers who stated that such boundary infringements on medical practitioner territory could stymy attempts at 'legitimation' and integration $[65,66]$. $\mathrm{T} \& \mathrm{CM}$ regulation was seen by policy-makers as a contractual bargain in return for T\&CM accepting practice restrictions aimed at protecting the public [66]. However, the possibility of practice restrictions arising from regulation concerned some Australian consumers [52], Australian T\&CM professional associations [52], and Australian, Canadian, and New Zealand T\&CM practitioners [50, 53, $71,73,77]$.

Other issues perceived by T\&CM providers were related to undue biomedical influence over practice or extra-professional regulatory oversight $[50,71,74,77]$. Some practitioners indicated this could negatively affect practice [73], others considered such influence would be overcome through regulation by allowing greater autonomy [69], a position also taken by some professional association representatives [54].
A related issue concerns practice misappropriation by untrained/undertrained practitioners from other branches of health care. While T\&CM practitioners perceived that regulation could prevent practice misappropriation [53, 69, 71], conventional medicine associations proposed co-optation as a way to maintain control of integration of T\&CM practices by conventional medical practitioners rather than non-medical T\&CM providers [67]. T\&CM practitioners [50, 71] and professional association representatives $[52,54]$ were concerned that external influences may lead to the diminution of traditional practice philosophies, as were consumers who lamented the potential loss of an intuitive practitioner approach due to regulation [52].

\section{Discussion}

This review provides the first known systematic examination of the contemporary empirical literature regarding stakeholder attitudes to the regulation of $\mathrm{T} \& \mathrm{CM}$ professions.

Of the 60 identified studies, six sought opinions from professional associations in Australia, Canada, and UK, two studies from Australia and Canada investigated education providers, and only two, both from Canada, canvassed the perceptions of policy-makers. The largely positive views of these stakeholders are tempered by the limited available research. The lack of research from many countries where $T \& C M$ is practised also limits the international generalisability of the findings. In particular, the views of policy-makers outside of Canada are yet to be determined and, consistent with other jurisdictional differences, may well be dissimilar to that seen in the present review. Scholars and commentators have long recognised the crucial role of policy-makers and policymaking in formally recognising $\mathrm{T} \& \mathrm{CM}$ professions. Policy-makers reported their priority of upholding the public interest while outlining a roadmap for regulation and integration. These were considered inducements in exchange for practice restrictions, which some T\&CM practitioners and professional associations considered unacceptable. Further research focussed explicitly on these stakeholder groups, particularly those of policymakers, is clearly needed to inform decisions regarding implementation of the WHO Traditional Medicine Strategy recommendations [9].

Another key finding from the review is that, consistent with regulatory policy provisions, the main focus of stakeholders outside of T\&CM professions was on public protection and raising inadequate training and practice standards. While these views were dominant among T\&CM practitioners as well, attention was also directed towards the professional benefits and disbenefits of regulation, suggesting that better communication regarding 
the purpose of regulation is needed. Previous research examining the impact of Chinese medicine regulation in the Australian state of Victoria supports the position that regulation enhances public safeguards with significant improvements in the management of consumer complaints and enforcement of professional standards after its inclusion in the statutory regulation scheme [106]. In addition, comparative examination of T\&CM and nonT\&CM professions in Australia's National Registration and Accreditation Scheme has been shown to work at least as well as conventional medical practitioner regulation $[107,108]$.

Our review found that some T\&CM practitioners perceived that professional associations adequately monitored and upheld professional standards obviating the need for regulation, a view contrary to stated consumer concerns. Examination of regulated and unregulated Australian T\&CM professions indicates that self-regulatory mechanisms are not as effective for improving public protection when using T\&CM services [109]. Furthermore, research on health care workforce regulatory frameworks and reforms emphasise the growing global focus on the public interest, a move away from self-regulatory governance models, and increased independence of complaints handling and oversight of disciplinary proceedings $[27,110]$.

As increasing T\&CM use is a largely consumer-driven phenomenon (given T\&CM is rarely integrated into public health systems), it is interesting that most consumers in this review stressed the need to regulate T\&CM practices, a finding consistent with research conducted in 1996 (prior to the date inclusion of this review) [111]. This temporal consistency of consumer opinion suggests a long-standing public preference for the independent governance of health practitioners, including T\&CM practitioners, who in this review were considered to require the same regulation as that governing conventional health practices. This is notable for two reasons. Firstly because consumer opinion regarding regulation persists despite the diversity of jurisdictions and T\&CM professions examined, and secondly because these results run counter to research identifying temporal considerations of stakeholder analysis as potentially limited [112].

Although a common critique of T\&CM regulation by some stakeholders is that regulation may grant these professions undue legitimacy, this view was not widely expressed in the identified studies. This finding, in addition to the worldwide reported rates of consumer use of [13-16], and trust in [113, 114] T\&CM practices suggests that the issue of professional legitimacy may be immaterial to a significant proportion of consumers and health professionals who may already view these practices as legitimate regardless of regulatory status [115].
Additionally, the relevance of established accountabilities and minimum standards of a profession for reasons of public safety and debates about professional legitimacy should be viewed as separate and divorced issues.

While the findings suggest support for T\&CM regulation across stakeholder groups, this may not necessarily indicate majority support. Support for or opposition to regulation of T\&CM providers is highly contextual and based on factors such as integration, marginalisation, perceived professionalisation, competition, as well as the specific type of therapy or practice. For example, support for regulation from conventional health practitioners was clearly not universal. Physiotherapists and manual therapists in the Netherlands were less supportive of regulation of chiropractors. Conventional medicine professionals in Korea were also less supportive of legislative governance of acupuncture, chiropractic, and traditional Chinese medicine. These differences may be partly explained by perceived inter-professional competition, as indicated by policy-makers and conventional medicine associations, and implied by physiotherapists and manual therapists in this review. The above examples suggest a clear competitive tension in terms of similar scopes of practice. Such competitive tensions have also been suggested to exist between conventional medicine and naturopathy in Australian, Canadian, and German studies [116-118]. One commentator has proposed that the care offered by $T \& C M$ practitioners could be used to alleviate conventional care workforce shortages [119], a role which is broadly supported (where appropriate) by the WHO [9]. Competition between medical and non-medical clinicians has also been a long-standing issue with respect to policy development and integration beyond T\&CM [120]. However, the degree to which T\&CM-specific issues influence standard issues associated with inter-professional competitive tensions, and consequently issues such as views towards regulation and scope of practice of another health profession requires further research before it can be confirmed.

In this review lack of regulation was cited by numerous stakeholders as exposing patients to direct and indirect risks. Yet some GPs indicated that well-trained T\&CM practitioners posed no risk of harm, and consumers indicated that T\&CM treatments should only be prescribed by qualified providers. The issue of harm, therefore, may be a function of the competence of the practitioner and the quality of T\&CM training $[121,122]$. Supporting this argument is the uncertainty regarding $T \& C M$ practitioner competence and training standards which was frequently reported by stakeholders in this review, including T\&CM practitioners. These, together with other nonhealth risks of T\&CM practices, have been extensively reviewed by researchers with many of these risks 
purported to be exacerbated by lack of regulation [123]. These findings suggest that risks to consumers posed by $\mathrm{T} \& \mathrm{CM}$ practices may be mitigated by appropriate regulatory mechanisms that promote greater public protections via appropriate standards and accountability, while ensuring that consumer choice is protected, and practices can be appropriately integrated where there is evidence of patient benefit. In support of this, naturopathic education standards have been found to vary internationally, with nations that have workforce regulatory frameworks in place reporting higher and more consistent education standards than those without regulation [32].

As distinct from previously mentioned conflicting opinions between T\&CM professions and consumers, there was also disagreement within the professions. The contrasting attitudes towards regulation between various T\&CM practitioners, and between practitioners and organisational representatives may potentially be driven by professional self-interest. Several studies highlighted the benefits to and concerns of T\&CM practitioners regarding regulation, revealing potential motivations of self-interest for attitudinal positions taken. 'Self-interest' may also be evident at the institutional level, particularly when organisations have been granted privileged roles within professions (such as through accredited or self-regulatory structures) that may be removed should those professions become statutorily regulated. This is suggested by previous examination, which has drawn attention to the resistance of much T\&CM regulation in Australia being led by professional associations with commercial interests in educational institutions that may be adversely affected by the higher educational standards imposed by regulation [52, 124]. This view accords with researchers of $\mathrm{T} \& \mathrm{CM}$ and non-T\&CM professions who contend that self-interest of education providers [125] and professional associations [126] are often incompatible with the public interest. As such, while T\&CM stakeholder perspectives should be considered important context for development of policy, they should not be elevated above other stakeholder perspectives (particularly consumer expectations), and ultimately regulatory decisions should be guided by public interest arguments, and jurisdictional, health system and professional needs, irrespective of T\&CM stakeholder views on regulation.

Despite a comprehensive search strategy, over twothirds of included studies were conducted in three jurisdictions; Australia, Canada, and New Zealand. Yet these jurisdictions represent only a small part of global T\&CM practice. High rates of consumer use of T\&CM practices are reported in sub-Saharan Africa [17], South America [18], the Arab states [19], Asia [16], India [20], and other world regions [16] where traditional medicine is integral to many cultural health practices and beliefs. These regions are implementing widespread T\&CM practitioner regulation [10] with little or no formal research examining stakeholder support, as evident from the review findings. Greater research focus is required into stakeholder opinions of regulation in these regions to ensure evidence-informed policy implementation efforts, and consistent application of regulatory measures across jurisdictions and practices.

Although most research on stakeholder attitudes and perceptions of T\&CM regulation has been conducted in Australia, Canada, and New Zealand, this appears to have had limited impact as these jurisdictions have been amongst the most hesitant to implement consistent workforce regulation policies across $\mathrm{T} \& \mathrm{CM}$ professions [10, 31]. A striking example comes from Australia where herbal medicine and naturopathy has consistent stakeholder support for regulation, as well as having regulation recommended by every Commonwealth and State government review of the issue since 2000. Yet these professions remain self-regulated almost 50 years after the first government review recommending they be statutorily regulated [127]. Australia, like many countries, has placed much of its T\&CM regulatory emphasis on product regulation, which the WHO considers is only part of the regulatory requirements it recommends to national governments [10]. This political inertia, described by some WHO member states as lack of 'political will' [10], also defies T\&CM evidence of efficacy and demonstrable public health arguments for regulation of T\&CM [9]. In addition to an increased global need for evidence to better inform regulatory decisions when implementing T\&CM regulation, governments should also be accountable for ensuring that evidence, where it exists, is used to inform appropriate T\&CM policy development. The most appropriate form of regulation for each health system, population, profession, and jurisdiction requires consideration based on evidence, and needs to be explored more explicitly. Our review indicates that regulation of TC\&M is not being conducted in an evidence-informed manner. The juxtaposition of jurisdictions generating stakeholder research which has not translated into regulatory policy with jurisdictions implementing regulation without corresponding stakeholder evidence may have significant policy implications and requires careful consideration from the research community engaging with this topic.

\section{Limitations}

This comprehensive review of the state of the current literature on the regulation of T\&CM professions comes with several limitations which should be considered when interpreting the findings. Firstly, one-third of studies was identified through hand and grey literature searching. 
This was likely due to varying definitions, terms and practices used across jurisdictions making a systematic search more difficult. The lack of consensus on T\&CM definitions resulted in considerable heterogeneity that was compounded by differences in jurisdictional definitions of conventional medicine versus T\&CM practices, and inconsistencies regarding regulation across stakeholder groups. The use of collective T\&CM terminology in some studies meant profession-specific data was not available. However, it is worth noting that T\&CM is, by definition, a subjective term defined by the health system in which care is offered and as such this variability is understandable. Critical appraisal of qualitative studies was also problematic because there was no identified fit-for-purpose tool for the predominantly descriptive nature of these studies. The included papers cover an extensive time period and variation in attitudinal perspectives due to increasing use of T\&CM practices may be expected over that time. However, temporal trends were generally not considered due to high heterogeneity across stakeholder groups as well as being considered of limited value in stakeholder analysis [112]. The results do not report on attitudes to the regulation of professions outside of T\&CM such as conventional providers or hospital staff. Nor do the results report the impact of regulation on institutional stakeholders such as health insurers, accreditors, and product manufacturers, or on the health care system generally. Due to these factors, we can only make claims about attitudinal responses to specific professions for a select group of included studies during a specific time period, with a call for greater research into health care workforce regulation in order to explicate these factors. Notwithstanding these limitations, this work provides the most extensive review of this topic to date and may be useful for researchers and policy-makers seeking to examine or implement appropriate $\mathrm{T} \& \mathrm{CM}$ regulation in line with WHO recommendations.

\section{Conclusions}

This systematic review identifies widespread consumer and practitioner support for the regulation of T\&CM professions. Significantly, consumers and practitioners from all branches of health care are calling for greater independent governance of, and accountably for unregulated T\&CM health care professions. The support for regulation derives from a need to safeguard the public by promoting practitioner competence through the establishment of professional and practice standards. Consumers, T\&CM practitioners, and conventional medicine practitioners comprised the vast majority of identified studies and their opinions regarding regulation are well represented in the literature. However, there is little research on the views of professional associations, education providers and policy-makers, and no published research on institutional stakeholders such as health insurers and accreditors. The available empirical evidence suggests stakeholders largely support regulation, with policy-makers expecting certain professional commutations which may not be acceptable to $T \& C M$ practitioners. In order to corroborate the conclusions of this review, further research is required from a broader range of jurisdictions using rigorous research methods. Determining attitudes across the breadth of health care stakeholders is a critical first step in offering insights into the barriers and enablers of regulation, developing relevant policy and practice recommendations, and informing appropriate policy change regarding $\mathrm{T} \& \mathrm{CM}$ professional regulation.

\section{Abbreviations}

GPs: General practitioners; T\&CM: Traditional and complementary medicine; WHO: World Health Organization.

\section{Acknowledgements}

Not applicable.

\section{Authors' contributions}

JC, JW and AS conceived of the design and methodology for this review. JC developed the review protocol and conducted the literature search with input and support from JW and AS. JC analysed and interpreted the results in conjunction with JW and AS. JC developed the initial draft of the manuscript and all authors contributed to writing, critically editing, revising, and approving the final manuscript. All authors read and approved the final manuscript.

\section{Funding}

Jenny Carè was supported by an Australian Government Research Training Program Scholarship while working on this manuscript. The funding body played no role in the study design; data collection, analysis, or interpretation; drafting or editing of the manuscript; or decision to submit the article for publication.

\section{Availability of data and materials}

All data generated or analysed during this study are included in this published article and key sources are cited.

\section{Declarations}

Ethics approval and consent to participate Not applicable.

Consent for publication

Not applicable.

\section{Competing interests}

$J C$ is a naturopath and current member of the Australian Register of Naturopaths and Herbalists, the Australian Natural Therapists Association, and the Naturopaths and Herbalists Association of Australia. JC is a board director of the Naturopaths and Herbalists Association of Australia, an appointment for which no remuneration is paid. AS is a director of the Australian Register of Naturopaths and Herbalists, an appointment for which no remuneration is paid. JW has no competing interests to declare.

\section{Author details}

${ }^{1}$ Australian Research Centre in Complementary and Integrative Medicine, University of Technology Sydney, Ultimo, NSW 2007, Australia. ${ }^{2}$ National Centre for Naturopathic Medicine, Southern Cross University, Lismore, NSW 2480, Australia. 
Received: 23 December 2020 Accepted: 1 March 2021

Published online: 29 March 2021

\section{References}

1. World Economic Forum: Value in healthcare: accelerating the pace of health system transformation. Switzerland. World Economic Forum. 2018. http://www3.weforum.org/docs/WEF_Value_in_Healthcare_ report_2018.pdf. Accessed 16 Oct 2020.

2. World Health Organization: Global status report on noncommunicable diseases 2010. Geneva. WHO. 2011. https://www.who.int/nmh/publi cations/ncd report full_en.pdf. Accessed 1 Oct 2020.

3. World Health Organization: Global strategy and action plan on ageing and health. Geneva. WHO. 2017. https://www.who.int/ageing/WHOGSAP-2017.pdf?ua=1. Accessed 17 Oct 2020.

4. Thomas S, Sagan A, Larkin J, Cylus J, Figueras J, Karanikolos M: Strengthening health systems resilience: key concepts and strategies. Policy Brief 26. Health Policy and Analysis. Regional Office for Europe. WHO 2020. https://www.euro.who.int/en/about-us/partners/observatory/ publications/policy-briefs-and-summaries/strengthening-health-syste ms-resilience-key-concepts-and-strategies-2020. Accessed 16 Oct 2020

5. World Health Organization: Global spending on health: a world in transition. (WHO/HIS/HGF/HFWorkingPaper/19.4). Licence: CC BY-NC-SA 3.0 IGO ed. Geneva. WHO. 2019. https://apps.who.int/iris/rest/bitstreams/ 1264564/retrieve\#: :text=both\%20out\%2Dof\%2Dpocket\%20and\% 20publicly\%20funded\&text=Two\%20years\%20into\%20the\%20Sustaina ble,US\%24\%207.6\%20trillion\%20in\%202016. Accessed 15 Oct 2020.

6. World Health Organization: Global strategy on human resources for health: workforce 2030. Geneva. WHO. 2016. https://www.who.int/publi cations/i/item/9789241511131. Accessed 15 Oct 2020.

7. World Health Organization: Classifying health workers: mapping occupations to the international standard classification. Geneva. WHO. 2010. https://www.who.int/hrh/statistics/workforce_statistics/en/. Accessed 16 Oct 2020.

8. Schmidt G: International classification of health workers - definitive guide. 2020. http://www.gregoryschmidt.ca/writing/international-class ification-of-health-workers. Accessed 16 Oct 2020.

9. World Health Organization: Traditional medicine strategy 2014-2023. Geneva. WHO. 2013. https://www.who.int/medicines/publications/ traditional/trm_strategy14_23/en/. Accessed 5 Sep 2020.

10. World Health Organization: Global report on traditional and complementary medicine 2019. Licence: CC BY-NC-SA 3.0 IGO ed. Geneva. WHO. 2019. https://apps.who.int/iris/bitstream/handle/10665/ 312342/9789241515436-eng.pdf?ua=1. Accessed 29 Aug 2020.

11. World Health Organization: Declaration of Alma-Ata, 1978. Regional Office for Europe. WHO. https://www.euro.who.int/en/publications/ policy-documents/declaration-of-alma-ata,-1978. Accessed 8 Feb 2021.

12. World Health Organization: Traditional medicine strategy 2002-2005. Geneva. WHO. 2002. https://apps.who.int/medicinedocs/pdf/s2297e/ s2297e.pdf. Accessed 5 Sep 2020.

13. Cooper KL, Harris PE, Relton C, Thomas KJ. Prevalence of visits to five types of complementary and alternative medicine practitioners by the general population: a systematic review. Complement Ther Clin Pract. 2013;19(4):214-20. https://doi.org/10.1016/j.ctcp.2013.06.006.

14. Frass M, Strassl RP, Friehs H, Müllner M, Kundi M, Kaye AD. Use and acceptance of complementary and alternative medicine among the general population and medical personnel: a systematic review. Ochsner J. 2012;12(1):45-56. https://www.ncbi.nlm.nih.gov/pmc/ articles/PMC3307506/.

15. Harris P, Cooper K, Relton C, Thomas K. Prevalence of complementary and alternative medicine (CAM) use by the general population: a systematic review and update. Int J Clin Pract. 2012;66(10):924-39. https://doi.org/10.1111/j.1742-1241.2012.02945.x.

16. Peltzer K, Pengpid S. Prevalence and determinants of traditional, complementary and alternative medicine provider use among adults from 32 countries. Chin J Integr Med. 2016;24(8):584-90. https://doi. org/10.1007/s11655-016-2748-y.

17. James P, Wardle J, Steel A, Adams J. Traditional, complementary and alternative medicine use in Sub-Saharan Africa: a systematic review. BMJ Glob Health. 2018;3(5):e000895. https://doi.org/10.1136/ bmjgh-2018-000895.

18. Brandão MGL, Acúrcio FA, Montemor RLM, Marlière LDP. Complementary/alternative medicine in Latin America: use of herbal remedies among a Brazilian metropolitan area population. J Complement Integr Med. 2006;3:1. https://doi.org/10.2202/1553-3840.1025.

19. Alrowais NA, Alyousefi NA. The prevalence extent of complementary and alternative medicine (CAM) use among Saudis. Saudi Pharm J. 2017;25(3):306-18. https://doi.org/10.1016/j.jsps.2016.09.009.

20. Maheshkumar K, Venugopal V, Poonguzhali S, Mangaiarkarasi N, Venkateswaran ST, Manavalan N. Trends in the use of yoga and naturopathy based lifestyle clinics for the management of non-communicable diseases (NCDs) in Tamilnadu South India. Clin Epidemiol Glob Health. 2020;8(2):647-51. https://doi.org/10.1016/j.cegh.2019.09.013.

21. Cooper RA, Laud P, Dietrich CL. Current and projected workforce of nonphysician clinicians. J Am Med Assoc. 1998;280(9):788-94. https://doi.org/10.1001/jama.280.9.788.

22. Cooper RA. Health care workforce for the twenty-first century: the impact of nonphysician clinicians. Annu Rev Med. 2001;52:51-61. https://pubmed.ncbi.nlm.nih.gov/11160767/.

23. Leach M. Profile of the complementary and alternative medicine workforce across Australia, New Zealand, Canada, United States and United Kingdom. Complement Ther Med. 2013;21(4):364-78. https:// doi.org/10.1016/j.ctim.2013.04.004.

24. Black J. Critical reflections on regulation. Austl J Leg Phil. 2002;27. http://www.academia.edu/download/7975081/disspaper4.pdf. Accessed 3 Sept 2020.

25. Freiberg A. Regulation in Australia. Sydney: Federation Press; 2017.

26. Selznick P. Focusing organizational research on regulation. In: Noll RG, editor. Regulatory policy and the social sciences. Berkeley: University of California Press; 1985. p. 363-7.

27. Yam CHK, Griffiths SM, Liu S, Wong ELY, Chung VCH, Yeoh EK. Medical regulation: ten key trends emerging from an international review. J Med Regul. 2016;102(1):16-27. https://doi.org/10.30770/2572-1852102.1.16.

28. Australian Health Practitioner Regulation Agency: Health Professions Accreditation Councils' Forum. Comparison of international accreditation systems for registered health professions. 2016. https://www.ahpra. gov.au/documents/default.aspx? record=WD17\%2F22815\&dbid=AP\& chksum =452xyoA39cjNlbE0cYfmTA\%3D\%3D. Accessed 28 Nov 2020.

29. Bartle I, Vass P. Self-regulation and the regulatory state: a survey of policy and practice. Research report 17. Bath: Centre for the Study of Regulation Industries, University of Bath School of Management; 2005.

30. Bourgeault IL, Grignon M. A comparison of the regulation of health professional boundaries across OECD countries. Eur J Comp Econ. 2013;10(2):199-223. http://ejce.liuc.it/Default.asp?tipo=articles\&ident ifier=ejce:18242979/2013/02/05.

31. Wardle JL, Sibbritt D, Broom A, Steel A, Adams J. Is health practitioner regulation keeping pace with the changing practitioner and healthcare landscape? An Australian perspective. Front Public Health. 2016. https://doi.org/10.3389/fpubh.2016.00091.

32. Dunn JM, Steel AE, Adams J, Lloyd I, De Groot N, Hausser T, et al. Characteristics of global naturopathic education, regulation, and practice frameworks: results from an international survey. BMC Complement Med Ther. 2021;21 (1):67. https://doi.org/10.1186/s12906-021-03217-1.

33. World Health Organization: Constitution of the World Health Organization, Chapter 1, Article 1. Forty-fifth edition, Supplement, October 2006 ed. Geneva. WHO. 2006. http://www.who.int/governance/eb/who_ constitution_en.pdf. Accessed 22 Nov 2020.

34. Turner RJ. Gower handbook of project management. 4th ed. Hampshire: Gower Publishing Limited; 2007.

35. Krosnick JA. Government policy and citizen passion: a study of issue publics in contemporary America. Polit Behav. 1990;12(1):59-92. https://doi.org/10.1007/BF00992332.

36. Burstein P. The impact of public opinion on public policy: a review and an agenda. Polit Res Q. 2003;56(1):29-40. https://doi.org/10.1177/10659 1290305600103.

37. Walt G, Gilson L. Reforming the health sector in developing countries: the central role of policy analysis. Health Policy Plan. 1994;9(4):353-70. https://doi.org/10.1093/heapol/9.4.353. 
38. Shea BJ, Hamel C, Wells GA, Bouter LM, Kristjansson E, Grimshaw $J$, et al. AMSTAR is a reliable and valid measurement tool to assess the methodological quality of systematic reviews. J Clin Epidemiol. 2009;62(10):1013-20. https://doi.org/10.1016/j.jclinepi.2008.10.009.

39. Moher D, Shamseer L, Clarke M, Ghersi D, Liberati A, Petticrew M, et al. Preferred reporting items for systematic review and meta-analysis protocols (PRISMA-P) 2015 statement. Syst Rev. 2015;4(1):1. https://doi. org/10.1186/2046-4053-4-1.

40. National Institute for Health Research: International prospective register of systematic reviews. http://www.crd.york.ac.uk/prospero. Accessed 1 Jun 2020.

41. Cochrane Complementary Medicine: Operational definition of complementary medicine. https:/cam.cochrane.org/operational-definitioncomplementary-medicine. Accessed 28 Mar 2020.

42. Zollman C, Vickers A. Complementary medicine in conventional practice. BMJ. 1999;319(7214):901-4. https://doi.org/10.1136/bmj.319.7214. 901.

43. Baer HA. The sociopolitical status of U.S. naturopathy at the dawn of the 21 st century. Med Anthropol Q. 2001;15(3):329-46. https://doi.org/10. 1525/maq.2001.15.3.329.

44. Hollenberg D. Uncharted ground: patterns of professional interaction among complementary/alternative and biomedical practitioners in integrative health care settings. Soc Sci Med. 2006;62(3):731-44. https:// doi.org/10.1016/j.socscimed.2005.06.030.

45. Mayberry JF. Review: Statutory regulation of invasive complementary therapies, such as hijama and acupuncture, is the only effective way of ensuring both patient safety and good practice. Med Leg J. 2018;86(1):23-31. https://doi.org/10.1177/0025817217734482.

46. Wardle J. The extraordinary complexities in defining complementary medicine. Adv Integr Med. 2017;4(3):89-90. https://doi.org/10.1016/j. aimed.2018.02.035.

47. Leach M. Natural, complementary, alternative: which term to use? Aust J Holist Nurs. 2003;10(1):46-9. https://pubmed.ncbi.nlm.nih.gov/12795 044/.

48. Lockwood C, Munn Z, Porritt K. Qualitative research synthesis: methodological guidance for systematic reviewers utilizing meta-aggregation. Int J Evid Based Healthc. 2015;13(3):179-87. https://doi.org/10.1097/ XEB.0000000000000062.

49. Hoy D, Brooks P, Woolf A, Blyth F, March L, Bain C, et al. Assessing risk of bias in prevalence studies: modification of an existing tool and evidence of interrater agreement. J Clin Epidemiol. 2012;65(9):934-9. https://doi.org/10.1016/j.jclinepi.2011.11.014.

50. Ijaz N, Boon H, Welsh S, Meads A. Supportive but "worried": perceptions of naturopaths, homeopaths and Chinese medicine practitioners through a regulatory transition in Ontario, Canada. BMC Complement Altern Med. 2015;15(1):1-13. https://doi.org/10.1186/ s12906-015-0846-6.

51. Wardle JL, Sibbritt DW, Adams J. Primary care practitioner perceptions and attitudes of complementary medicine: a content analysis of freetext responses from a survey of non-metropolitan Australian general practitioners. Prim Health Care Res Dev. 2018;19(3):246-55. https://doi. org/10.1017/S1463423617000664.

52. Lin V, Bensoussan A, Myers S, McCabe P, Cohen M, Hill S, et al. The practice and regulatory requirements of naturopathy and western herbal medicine. Bundoora, Victoria: La Trobe University School of Public Health; 2005. https://www.yourhealthyourchoice.com.au/wp-conte nt/uploads/2018/04/Vic-govt-Practice-and-Regulatory-Requirementsof-Naturopathy-Western-Herbal-Medicine-2005.pdf. Accessed 20 May 2020.

53. Boon H, Welsh S, Kelner M, Wellman B. CAM practitioners and the professionalisation process: a Canadian comparative case study. In: Tovey P, Easthope G, Adams J, editors. The mainstreaming of complementary and alternative medicine: studies in social context. London: Routledge; 2004. p. 123-39.

54. Clarke DB, Doel MA, Segrott J. No alternative? The regulation and professionalization of complementary and alternative medicine in the United Kingdom. Health Place. 2004;10(4):329-38. https://doi.org/10. 1016/j.healthplace.2004.08.001.

55. Ericksen-Pereira W, Roman NV, Swart R. The effect of legislation on the treatment practices and role of naturopaths in South Africa. BMC
Complement Med Ther. 2020;20(1):139. https://doi.org/10.1186/ s12906-020-02916-5.

56. Gyasi RM, Poku AA, Boateng S, Amoah PA, Mumin AA, Obodai J, et al. Integration for coexistence? Implementation of intercultural health care policy in Ghana from the perspective of service users and providers. J Integr Med. 2017;15(1):44-55. https://doi.org/10.1016/s2095-4964(17) 60312-1.

57. Malhotra V, Harnett J, Wong K, Saini B. The perspectives of Australian naturopaths about providing health services for people with sleep disorders. Behav Sleep Med. 2020. https://doi.org/10.1080/15402002. 2020.1751164.

58. Smith D. Perceptions and benefits of, and barriers to, degree-based education for massage therapy [Doctoral dissertation]. Dunedin, New Zealand: University of Otago; 2015. http://hdl.handle.net/10523/5610. Accessed 26 May 2020.

59. James PB, Wardle J, Steel A, Adams J, Bah AJ, Sevalie S. Traditional and complementary medicine use among Ebola survivors in Sierra Leone: a qualitative exploratory study of the perspectives of healthcare workers providing care to Ebola survivors. BMC Complement Med Ther. 2020;20(1):137. https://doi.org/10.1186/s12906-020-02931-6.

60. Steel A, Diezel H, Frawley J, Wardle J, Adams J. Providing maternity care from outside the system: perspectives of complementary medicine practitioners. J Interprof Care. 2020. https://doi.org/10.1080/13561820. 2020.1711717.

61. Barnes J, Butler R. Community pharmacists'views on the regulation of complementary medicines and complementary-medicines practitioners: a qualitative study in New Zealand. Int J Pharm Pract. 2018;26(6):485-93. https://doi.org/10.1111/ijpp.12428.

62. Flower A, Winters D, Bishop FL, Lewith $\mathrm{G}$. The challenges of treating women with recurrent urinary tract infections in primary care: a qualitative study of GPs' experiences of conventional management and their attitudes towards possible herbal options. Prim Health Care Res Dev. 2015;16(6):597-606. https://doi.org/10.1017/\$1463423615000201.

63. Gilmour J, Kelner M, Wellman B. Opening the door to complementary and alternative medicine: self-regulation in Ontario. Law Policy. 2002;24(2):149-74. https://doi.org/10.1111/1467-9930.00132.

64. Jarvis A, Perry R, Smith D, Terry R, Peters S. General practitioners' beliefs about the clinical utility of complementary and alternative medicine. Prim Health Care Res Dev. 2015;16(3):246-53. https://doi.org/10.1017/ S146342361400022X.

65. Kelly M, Hardwick K, Moritz S, Kelner M, Rickhi B, Quan H. Towards integration: opinions of health policy makers on complementary and alternative medicine. Evid Based Integrative Med. 2005;2(2):79-86. https://doi.org/10.2165/01197065-200502020-00003.

66. Kelner M, Wellman B, Boon H, Welsh S. The role of the state in the social inclusion of complementary and alternative medical occupations. Complement Ther Med. 2004;12(2-3):79-89. https://doi.org/10.1016/j. ctim.2004.09.002.

67. Kelner M, Wellman B, Boon H, Welsh S. Responses of established healthcare to the professionalization of complementary and alternative medicine in Ontario. Soc Sci Med. 2004;59(5):915-30.

68. Kelner M, Wellman B, Welsh S, Boon H. How far can complementary and alternative medicine go? The case of chiropractic and homeopathy. Soc Sci Med. 2006;63(10):2617-27. https://doi.org/10.1016/j.socscimed. 2006.07.005.

69. Wardle JL, Adams J, Chi-Wai L, Steel AE. Current challenges and future directions for naturopathic medicine in Australia: a qualitative examination of perceptions and experiences from grassroots practice. BMC Complement Altern Med. 2013;13(1):15-24. https://doi.org/10.1186/ 1472-6882-13-15.

70. Welsh S, Kelner M, Wellman B, Boon H. Moving forward? Complementary and alternative practitioners seeking self-regulation. Sociol Health IIIn. 2004;26(2):216-41. https://doi.org/10.1111/j.1467-9566.2004. 00387.x.

71. Canaway R. A culture of dissent: Australian naturopaths perspectives on practitioner, regulation. Complement Health Pract Rev. 2009;14(3):13652. https://doi.org/10.1177/1533210109360308.

72. Cavaco AM, Arslan M, Azar S. Informing the homeopathic practice for Turkish pharmacists: reviewing the example of Portuguese community pharmacies. Homeopathy. 2017;106(2):93-102. https://doi.org/10. 1016/j.homp.2017.02.003. 
73. Bensoussan A, Myers SP, Wu SM, O'Connor K. Naturopathic and Western herbal medicine practice in Australia—a workforce survey. Complement Ther Med. 2004;12(1):17-27. https://doi.org/10.1016/j.ctim.2004. 01.001.

74. Braun LA, Spitzer O, Tiralongo E, Wilkinson JM, Bailey M, Poole SG, et al. Naturopaths and Western herbalists' attitudes to evidence, regulation, information sources and knowledge about popular complementary medicines. Complement Ther Med. 2013;21 (1):58-64. https://doi.org/ 10.1016/j.ctim.2012.11.008.

75. Dooley M, Braun L, Poole S, Bailey M, Spitzer O, Tiralongo E, et al. Investigating the integration of complementary medicines in community pharmacy practice. Melbourne: Australian Government Department of Health and Ageing, and The Pharmacy Guild of Australia; 2010. http:// 6cpa.com.au/wp-content/uploads/The-Integration-of-Complement ary-Medicines-in-Community-Pharmacy-Practice-Full-Final-Report.pdf. Accessed 26 Jun 2020.

76. Emslie M, Campbell MK, Walker K. Changes in public awareness of, attitudes to, and use of complementary therapy in North East Scotland: surveys in 1993 and 1999. Complement Ther Med. 2002;10(3):148-53. https://doi.org/10.1016/S0965229902000663.

77. Flatt J, Hahn S. Naturopathic and Western herbal medicine practitioners' perception of proposed changes to regulatory status in New Zealand: an exploratory survey. Int J Naturopathic Med. 2013;6. https://intjnm. $\mathrm{com} /$ naturopathic-and-western-herbal-medicine-practitioners-perce ption-of-proposed-changes-to-regulatory-status-in-new-zealand-anexploratory-survey/. Accessed 3 Sept 2020.

78. Parker A. Ontario homeopathic practitioner survey: are homeopaths prepared to be regulated? [Master's thesis]. Toronto, Canada: University of Toronto; 2013. https://tspace.library.utoronto.ca/bitstream/1807/ 70023/1/Aalia_Parker_201311_MSc_thesis.pdf. Accessed 20 Jun 2020.

79. Zhang AL, Story DF, Lin V, Vitetta L, Xue CC. A population survey on the use of 24 common medicinal herbs in Australia. Pharmacoepidemiol Drug Saf. 2008;17(10):1006-13. https://doi.org/10.1002/pds.1610.

80. McCabe P. Education in naturopathy and western herbal medicine in Australia: results of a survey of education providers. Complement Ther Clin Pract. 2008;14(3):168-75. https://doi.org/10.1016/j.ctcp.2008.02. 002.

81. Chaterji R, Tractenberg RE, Amri H, Lumpkin M, Amorosi SBW, Haramati A. A large-sample survey of first-and second-year medical student attitudes toward complementary and alternative medicine in the curriculum and in practice. Altern Ther Health Med. 2007;13(1):30-5.

82. Cottingham P, Adams J, Vempati R, Dunn J, Sibbritt D. The characteristics, experiences and perceptions of registered massage therapists in New Zealand: results from a national survey of practitioners. Int J Ther Massage Bodywork. 2018;11(2):11-24. https://doi.org/10.3822/ijtmb. v11i2.385.

83. Evans A, Duncan B, McHugh P, Shaw J, Wilson C. Inpatient's use, understanding, and attitudes towards traditional, complementary and alternative therapies at a provincial New Zealand hospital. N Z Med J. 2008;121(1278):21-34. https://pubmed.ncbi.nlm.nih.gov/18670472/.

84. Harris IM, Kingston RL, Rodriguez R, Choudary V. Attitudes towards complementary and alternative medicine among pharmacy faculty and students. Am J Pharm Educ. 2006. https://doi.org/10.5688/aj700 6129

85. Langworthy JM, Smink RD. Chiropractic through the eyes of physiotherapists, manual therapists, and osteopaths in The Netherlands. J Altern Complement Med. 2000;6(5):437-43. https://doi.org/10.1089/ acm.2000.6.437.

86. Livingston CJ, Krass I, Li GQ. Factors predicting the recommendations of general practitioners on herbal therapies and dietary supplements to patients. J Complement Integr Med. 2010;7:1. https://doi.org/10.2202/ 1553-3840.1370.

87. Montbriand M. Alternative therapies. Health professionals' attitudes. Can Nurse. 2000;96(3):22-6. https://pubmed.ncbi.nlm.nih.gov/11143 $657 /$.

88. Morin C, Desrosiers J, Gaboury I. Descriptive study of interprofessional collaboration between physicians and osteopaths for the pediatric population in Quebec, Canada. BMC Health Serv Res. 2017;17:1-8. https://doi.org/10.1186/s12913-017-2717-y.
89. Price J, White A. The use of acupuncture and attitudes to regulation among doctors in the UK-a survey. Acupunct Med. 2004;22(2):72-4. https://doi.org/10.1136/aim.22.2.72.

90. Semple SJ, Hotham E, Rao D, Martin K, Smith CA, Bloustien GF. Community pharmacists in Australia: barriers to information provision on complementary and alternative medicines. Pharm World Sci. 2006;28(6):366-73. https://doi.org/10.1007/s11096-006-9058-y.

91. Taylor M. Patients' and general practitioners' attitudes towards complementary medicine in Wanganui, New Zealand. N Z Fam Physician. 2003;30(2):102-7.

92. Tsai P-S, Lee P-H, Wang M-Y. Demographics, training, and practice patterns of practitioners of folk medicine in Taiwan: a survey of the Taipei metropolitan area. J Altern Complement Med. 2008;14(10):1243-8. https://doi.org/10.1089/acm.2008.0316.

93. Xue C, Clifton L, Karagiannis J, Li C, Yang A, Zhang L, et al. Public attitudes towards Chinese medicine in Melbourne, Australia. J Complement Integr Med. 2005;2(1):1-11. https://doi.org/10.2202/1553-3840. 1024.

94. Zhang A. Complementary and alternative medicine use in Australia: a national population-based study [Doctoral dissertation]. Victoria, Australia: RMIT University; 2006. http://www.academia.edu/download/ 49719126/Zhang.pdf. Accessed 4 Jun 2020.

95. Al Mansour MA, Al-Bedah AM, AlRukban MO, Elsubai IS, Mohamed EY, El Olemy AT, et al. Medical students' knowledge, attitude, and practice of complementary and alternative medicine: a pre-and post-exposure survey in Majmaah University, Saudi Arabia. Adv Med Educ Pract. 2015;6:407-20. https://doi.org/10.2147/AMEP.S82306.

96. Cottingham P, Adams J, Vempati R, Dunn J, Sibbritt D. The characteristics, experiences and perceptions of naturopathic and herbal medicine practitioners: results from a national survey in New Zealand. BMC Complement Altern Med. 2015;15(1):1-11. https://doi.org/10.1186/ s12906-015-0616-5.

97. Cottingham P, Adams J, Vempati R, Dunn J, Sibbritt D. The characteristics, experiences and perceptions of homeopaths in New Zealand: results from a national survey of practitioners. Homeopathy. 2017;106(1):11-7. https://doi.org/10.1016/j.homp.2016.12.003.

98. Poynton L, Dowell A, Dew K, Egan T. General practitioners'attitudes toward (and use of) complementary and alternative medicine: a New Zealand nationwide survey. N Z Med J. 2006;1 19(1247):35-44. https:// pubmed.ncbi.nlm.nih.gov/17195854/.

99. Tiralongo E, Braun L, Wilkinson J, Spitzer O, Bailey M, Poole S, et al. Exploring the integration of complementary medicines into Australian pharmacy practice with a focus on different practice settings and background knowledge. J Complement Integr Med. 2010;7(1):1-21. https:// doi.org/10.2202/1553-3840.1411.

100. Yu J-S, Kim C-B, Kim K-K, Lee J-E, Kim M-Y. Behaviors of providers of traditional Korean medicine therapy and complementary and alternative medicine therapy for the treatment of cancer patients. J Pharmacopuncture. 2015;18(1):27-35. https://doi.org/10.3831/KPI.2015.18.003.

101. Cohen MM, Penman S, Pirotta M, da Costa C. The integration of complementary therapies in Australian general practice: results of a national survey. J Altern Complement Med. 2005;11(6):995-1004. https://doi. org/10.1089/acm.2005.11.995.

102. El-Olemy AT, Radwan NM, Shihab NSE, Dawood WM. Knowledge, attitudes, and practices of non-medical students in traditional and complementary medicine in Gharbiya governorate, Egypt: a crosssectional study. Complement Med Res. 2014;21(5):314-9. https://doi. org/10.1159/000367979.

103. Hall K, Giles-Corti B. Complementary therapies and the general practitioner. A survey of Perth GPs. Aust Fam Physician. 2000;29(6):602-6.

104. Poreddi V, Thiyagarajan S, Swamy P, Ramachandra GS, Thimmaiah R, et al. Nursing students attitudes and understanding of complementary and alternative therapies: an Indian perspective. Nurs Educ Perspect. 2016;37(1):32-7.

105. Lin V, McCabe P, Bensoussan A, Myers S, Cohen M, Hill S, et al. The practice and regulatory requirements of naturopathy and western herbal medicine in Australia. Risk Manag Healthc Policy. 2009;2:21-33. https:// doi.org/10.2147/RMHP.S4652.

106. Lin V, Gillick D. Does workforce regulation have the intended effect? The case of Chinese medicine practitioner registration. Aust Health Rev. 2011;35(4):455. https://doi.org/10.1071/AH10869. 
107. Sibbritt D, Kaye M, Millbank J, Stuhmcke A, Wardle J, Karpin I. How are complementary health professions regulated in Australia? An examination of complementary health professions in the national registration and accreditation scheme. Complement Ther Med. 2018;37:6-12. https://doi.org/10.1016/j.ctim.2017.12.012.

108. Millbank J, Kaye M, Stuhmcke A, Sibbritt D, Karpin I, Wardle J. Complementary health practitioners disciplined for misconduct in Australia 2010-2016. J Law Med. 2017;24(4):788-802.

109. Wardle J. Holding unregistered health practitioners to account: an analysis of current regulatory and legislative approaches. J Law Med. 2014;22(2):350-75. https://pubmed.ncbi.nlm.nih.gov/25715537/.

110. Leslie K, Nelson S, Deber R, Gilmour J. Policy tensions in regulatory reform: changes to regulation of health professions in Australia, the United Kingdom, and Ontario. Canada J Nurs Regul. 2018;8(4):32-42. https://doi.org/10.1016/s2155-8256(17)30180-1.

111. Emslie M, Campbell M, Walker K. Complementary therapies in a local healthcare setting. Part I: Is there real public demand? Complement Ther Med. 1996;4(1):39-42. https://doi.org/10.1016/S0965-2299(96) 80054-9.

112. Brugha R, Varvasovszky Z. Stakeholder analysis: a review. Health Policy Plan. 2000;15(3):239-46. https://doi.org/10.1093/heapol/15.3.239.

113. Emmerton L, Fejzic J, Tett SE. Consumers' experiences and values in conventional and alternative medicine paradigms: a problem detection study (PDS). BMC Complement Altern Med. 2012;12:39. https://doi.org/ 10.1186/1472-6882-12-39.

114. Foley $\mathrm{H}$, Steel A, Adams J. Perceptions of person-centred care amongst individuals with chronic conditions who consult complementary medicine practitioners. Complement Ther Med. 2020;52:102518. https://doi. org/10.1016/j.ctim.2020.102518.

115. Wardle J, Gallego-Perez D, Chung V, Adams J. Traditional, complementary and integrative medicine: policy, legal and regulatory perspectives. In: Adams J, editor. Public health and health services research in traditional, complementary and integrative health care: international perspectives. London: World Scientific Publishing Europe Ltd; 2019. p. 57-78.

116. Joos S, Musselmann B, Miksch A, Rosemann T, Szecsenyi J. The role of complementary and alternative medicine (CAM) in Germany-a focus group study of GPs. BMC Health Serv Res. 2008;8(1):127. https://doi.org/ 10.1186/1472-6963-8-127.

117. Verhoef M, Boon H, Mutasingwa D. The scope of naturopathic medicine in Canada: an emerging profession. Soc Sci Med. 2006;63(2):409-17. https://doi.org/10.1016/j.socscimed.2006.01.008.
118. Wardle J, Sibbritt D, Adams J. The interface with naturopathy in rural primary health care: a survey of referral practices of general practitioners in rural and regional New South Wales, Australia. BMC Complement Altern Med. 2014;14:238. https://doi.org/10.1186/1472-6882-14-238.

119. Leach MJ. Using role substitution to address the health workforce shortage and to facilitate integration? J Complement Integr Med. 2012. 9(1):Article 30. https://doi.org/10.1515/1553-3840.1519.

120. Grumbach K, Coffman J. Physicians and nonphysician clinicians: complements or competitors? JAMA. 1998;280(9):825-6. https://doi.org/10. 1001/jama.280.9.825.

121. Pirotta MV, Cohen MM, Kotsirilos V, Farish SJ. Complementary therapies: have they become accepted in general practice? Med J Aust. 2000;172(3):105-9. https://doi.org/10.5694/j.1326-5377.2000.tb127 932.x.

122. Wardle J, Steel A, Adams J. A review of tensions and risks in naturopathic education and training in Australia: a need for regulation. J Altern Complement Med. 2012;18(4):363-70. https://doi.org/10.1089/ acm.2011.0480.

123. Wardle J, Adams J. Indirect and non-health risks associated with complementary and alternative medicine use: an integrative review. Eur J Integr Med. 2014;6(4):409-22. https://doi.org/10.1016/j.eujim.2014.01. 001.

124. Baer H. Complementary medicine in Australia and New Zealand. Its popularisation, legitimation and dilemmas. London: Routledge; 2016.

125. Aldridge $S$. The regulation of health professionals: an overview of the British Columbia experience. J Med Imaging Radiat Sci. 2008;39(1):4-10. https://doi.org/10.1016/j.jmir.2008.01.001.

126. Young K. Politics ahead of patients: the battle between medical and chiropractic professional associations over the inclusion of chiropractic in the American medicare system. Bull Can Hist Med. 2019;36(2):381. https://doi.org/10.3138/cbmh.330-022019.

127. Victorian Parliament Joint Select Committee: Report from the osteopathy, chiropractic and naturopathy committee. Melbourne. C. H. Rixon, Government Printer. 1975. https://www.parliament.vic.gov.au/vufind/ Record/7450. Accessed 24 Jun 2020.

\section{Publisher's Note}

Springer Nature remains neutral with regard to jurisdictional claims in published maps and institutional affiliations.
Ready to submit your research? Choose BMC and benefit from:

- fast, convenient online submission

- thorough peer review by experienced researchers in your field

- rapid publication on acceptance

- support for research data, including large and complex data types

- gold Open Access which fosters wider collaboration and increased citations

- maximum visibility for your research: over 100M website views per year

At BMC, research is always in progress.

Learn more biomedcentral.com/submissions 